\title{
Gene cassette knock-in in mammalian cells and zygotes by enhanced MMEJ
}

\author{
Tomomi Aida ${ }^{1,2,7^{*} \dagger}$, Shota Nakade ${ }^{5 \dagger}$, Tetsushi Sakuma ${ }^{5^{*}}$, Yayoi Izu ${ }^{4}$, Ayu Oishi ${ }^{5,8}$, Keiji Mochida ${ }^{5}$, Harumi Ishikubo ${ }^{1,2}$, \\ Takako Usami ${ }^{2}$, Hidenori Aizawa ${ }^{1,6}$, Takashi Yamamoto ${ }^{5^{*}}$ and Kohichi Tanaka ${ }^{1,3^{*}}$ (D)
}

\begin{abstract}
Background: Although CRISPR/Cas enables one-step gene cassette knock-in, assembling targeting vectors containing long homology arms is a laborious process for high-throughput knock-in. We recently developed the CRISPR/Cas-based precise integration into the target chromosome (PITCh) system for a gene cassette knock-in without long homology arms mediated by microhomology-mediated end-joining.

Results: Here, we identified exonuclease 1 (Exo1) as an enhancer for PITCh in human cells. By combining the Exo 1 and PITCh-directed donor vectors, we achieved convenient one-step knock-in of gene cassettes and floxed allele both in human cells and mouse zygotes.
\end{abstract}

Conclusions: Our results provide a technical platform for high-throughput knock-in.

Keywords: MMEJ, CRISPR/Cas, Gene cassette, Reporter, Flox, Knock-in, Mouse, Exo1, High throughput, Cloning-free

\section{Background}

Knock-in mice carrying functional gene cassettes have provided invaluable opportunities for in vivo functional analysis of genes and cells in mammalian organisms [1]. Despite the recent rapid displacement of conventional gene-targeting technology in embryonic stem cells [2] by CRISPR/Cas-mediated genome editing [3-5] in mouse zygotes [6-10], the cumbersome task of targeting vector construction containing a gene cassette flanked by long homology arms (generally longer than several kilobases for each) corresponding to every target loci remains unchanged $[11,12]$. This limits the feasibility of CRISPR/Cas-mediated, large-scale, highthroughput generation $[13,14]$ of knock-in mice carrying functional gene cassettes and limits the convenience and availability of the CRISPR/Cas system for mammalian organisms $[1,15]$.

\footnotetext{
* Correspondence: aidat@mit.edu; tetsushi-sakuma@hiroshima-u.ac.jp: tybig@hiroshima-u.ac.jp; tanaka.aud@mri.tmd.ac.jp

${ }^{\dagger}$ Equal contributors

'Laboratory of Molecular Neuroscience, Medical Research Institute (MRI), Tokyo Medical and Dental University (TMDU), 1-5-45, Yushima, Bunkyo, Tokyo 113-8510, Japan

${ }^{5}$ Department of Mathematical and Life Sciences, Graduate School of Science, Hiroshima University, 1-3-1, Kagamiyama, Higashi-Hiroshima, Hiroshima 739-8526, Japan

Full list of author information is available at the end of the article
}

The homologous recombination (HR)-mediated repair of DNA double-strand breaks (DSBs) induced by CRISPR/ Cas has been used to knock-in the gene cassettes in endogenous target genomic loci in mouse zygotes $[6,8,10]$. However, the DSBs are primarily repaired through nonhomologous end joining (NHEJ) in mammalian cells, and thus the frequency of HR-mediated repair is intrinsically low [16]. The efficiencies of HR-mediated gene cassette knock-in in mice are around $10-20 \%$, much less than that (up to 100\%) of NHEJ-mediated gene knockout [11, 12]. We recently reported the cloning-free CRISPR/Cas system, which is based on the Cas9 protein and chemically synthesized dual-RNAs (crispr RNA [crRNA] and transactivating crRNA [tracrRNA]) instead of broadly used single-guide RNA (sgRNA, a chimeric molecule of crRNA and tracrRNA), facilitates HR-mediated gene cassette knock-in in mice by up to 50\% [8]. The technological development of CRISPR/Cas-mediated gene cassette knock-in in mice is just beginning, and much improvement is required.

A major alternative pathway for DSB repair, named microhomology-mediated end joining (MMEJ), joins the ends of DSBs by utilizing microhomology for the alignment of broken ends, leading to deletions at the site of the DSBs [17]. Interestingly, the microhomologies were frequently found in the majority of repaired sites of the DSBs induced by CRISPR/Cas in mice [18] and human 
cells (more than half of the deletions [19]). Taking advantage of the high frequency of MMEJ, we recently developed the highly efficient and convenient CRISPR/Cas or transcription activator-like effector nuclease (TALEN)based precise integration into the target chromosome (PITCh) system that harnesses MMEJ to knock-in a gene cassette into target genomic loci with extremely short ( $\leq 40 \mathrm{bp}$ ) microhomologies in cultured cells [20-22], silkworms [20], frogs [20], and zebrafish [23]. In addition to its high efficiency, the PITCh system has the potential to omit the laborious steps of constructing a targeting vector for gene cassette knock-in by the use of microhomologies. The PITCh system may be a key technology for feasible, largescale, knock-in projects [14] to generate thousands of mice expressing enhanced green fluorescent protein (EGFP) or Cre recombinase under the control of an endogenous promoter, similar to the gene expression nervous system atlas (GENSAT) project for bacterial artificial chromosome transgenic mice [13]; however, its validity in mammalian organisms has not been examined. Here, we show the efficient and highly convenient PITCh-based knock-in strategy in mice by the combination of an MMEJ-directed simplified donor vector, overexpression of the MMEJ-enhancing factor, and the cloning-free CRISPR/Cas system.

\section{Results}

\section{Generation of knock-in mice carrying a gene cassette by} the PITCh system

To test the PITCh system in mouse zygotes, we chose the $A c t b$ locus [24, 25] where we previously knocked-in a 2.5$\mathrm{kb}$ gene cassette by the cloning-free CRISPR/Cas system with a conventional targeting vector containing 2-kb homology arms [8]. In the present study, we knocked-in a 5-kb TetO-FLEX-hM3Dq/mCherry gene cassette (TetO operator [tetO] sequences followed by inverted Gq-coupled human M3 muscarinic DREADD (designer receptors exclusively activated by designer drug, hM3Dq)/mCherry flanked by two pairs of loxP and loxP2722 [FLEX switch]) to the Actb locus (Fig. 1a) [1, 8, 15, 26]. Since linearization of the donor is required for gene cassette knock-in by the PITCh system, we designed a synthetic guide RNA sequence $(g R N A-s 1)$ as the universal guide RNA that shares no sequence homology with the genomic DNA of several mammalian species, including human and mouse [21]. We constructed PITCh-directed donor vectors (PITChdonor) [21] containing a 5-kb TetO-FLEX-hM3Dq/ mCherry cassette flanked by 40 -bp left and right microhomologies corresponding to $800 \mathrm{bp}$ downstream of the $A c t b$ polyA signal and gRNA-s1 crRNA target sequences (Fig. 1a). The PITCh donor was efficiently digested by in vitro digestion assay (IDA) with $g R N A-s 1$ crRNA, tracrRNA, and Cas9 protein (Additional file 1: Figure S1).

We then injected the circular PITCh donor together with chemically synthesized Actb and $g R N A-s 1$ crRNAs and tracrRNA, and Cas9 protein into one-cell-stage mouse zygotes (Fig. 1b) [8]. We obtained 25 newborns, and screened their tail genomic DNA by PCR with three different primer sets (Fig. 1a) to identify knock-in mice (Fig. 1c, d and Additional file 1: Figure S2). We found three knock-in mice defined by triple PCR positive carrying a TetO-FLEX-hM3Dq/mCherry cassette at the Actb locus (Fig. 1c, d and Additional file 1: Figure S2). Knock-in efficiency was 12\% (Fig. 1d). We also found two partial knock-in mice defined by double PCR positive for LF + LR and IF + IR carrying a part of the cassette at the Actb locus (mice \#10 and \#18 in Additional file 1: Figures S2). Next, we sequenced the PCR products of the left and right boundaries between Actb and the TetO-FLEX-hM3Dq/mCherry cassette and found the precise knock-in of the cassette we designed (Fig. 1e). Although left boundaries were precisely knocked-in in two partial knock-in mice, we could not determine their right boundaries (data not shown). We also sequenced the PCR products of non-knock-in Actb alleles amplified with LF and RR primers. These alleles were modified by NHEJ in $92 \%$ of the newborn mice (Fig. 1d and Additional file 1: Figure S3). Collectively, the knock-in mice carrying a gene cassette could be generated by the PITCh system in combination with cloning-free CRISPR/Cas system. However, its efficiency (12\%, Fig. 1d) was much lower than that of our previous study (45.5\%, [8]), which was accomplished by the combination of a conventional targeting vector with long homology arms and the cloning-free CRISPR/Cas system, although the length of knock-in cassette in this study was larger than that of previous report ( $5 \mathrm{~kb}$ vs. $2.5 \mathrm{~kb}$ ).

\section{Genetic screening of MMEJ enhancer}

To enhance the efficiency of the MMEJ-mediated gene cassette knock-in, we conducted genetic screening to identify genes that enhance MMEJ. We constructed a fluorescent reporter system to detect MMEJ-mediated repair of DSBs, similar to the previous report [27]. The reporter plasmid expressing inactive (out-of-frame) EGFP was split by a CRISPR target sequence containing two tandem microhomologies under the control of the CMV promoter (Fig. 2a). When the DSBs in the reporter plasmid induced by CRISPR are repaired through MMEJ between two microhomologies, functional in-frame EGFP is reconstituted and EGFP fluorescence is recovered (Fig. 2a). We chose 13 candidate genes from among those involved in DSB repair pathways (Exonuclease 1 [Exo1], DNA Ligase 3 [Lig3], Poly (ADP-Ribose) Polymerase 1 [PARP1], Nijmegen Breakage Syndrome protein 1 [NBS1], Flap structure-specific Endonuclease 1 [FEN1], Bloom syndrome RecQ like helicase [BLM], Meiotic Recombination 11A [MRE11A], RAD51, RAD52, Three Prime Repair Exonuclease 2 [TREX2], dominant-negative 


\section{a Wildtype}

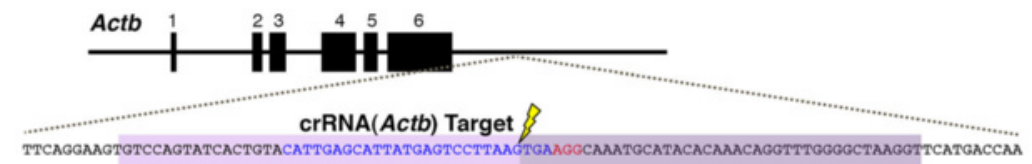

40-bp MH

40-bp MH

GTGCTTCGATATCGATCGTTTGGGTCCAGTATCACTGTACATTGaGCATTATGAGTCCTTAAGAC crRNA(gRNA-s1) Target

Target

PITCh-Donor

Teto LoxP/2722 LoxP/2722

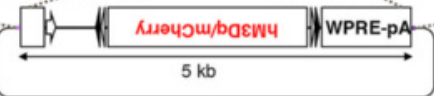

MMEJ-mediated knock-in

Knock-in

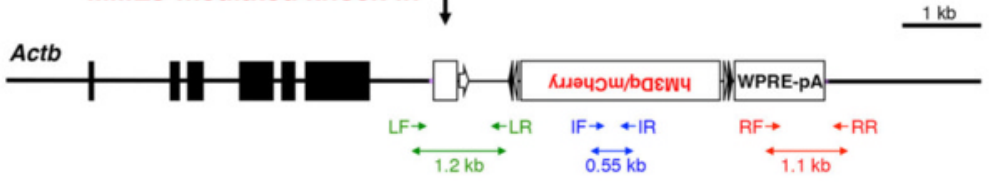

b

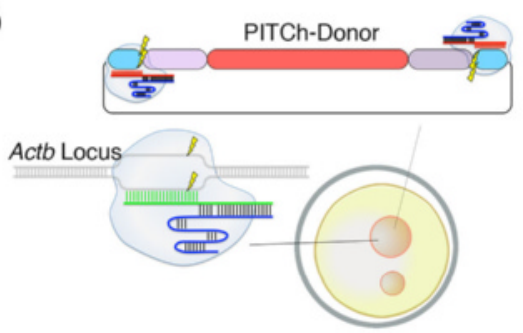

Transfer

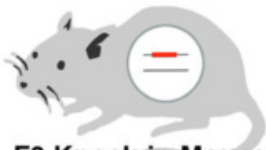

Fo Knock-in Mouse
C

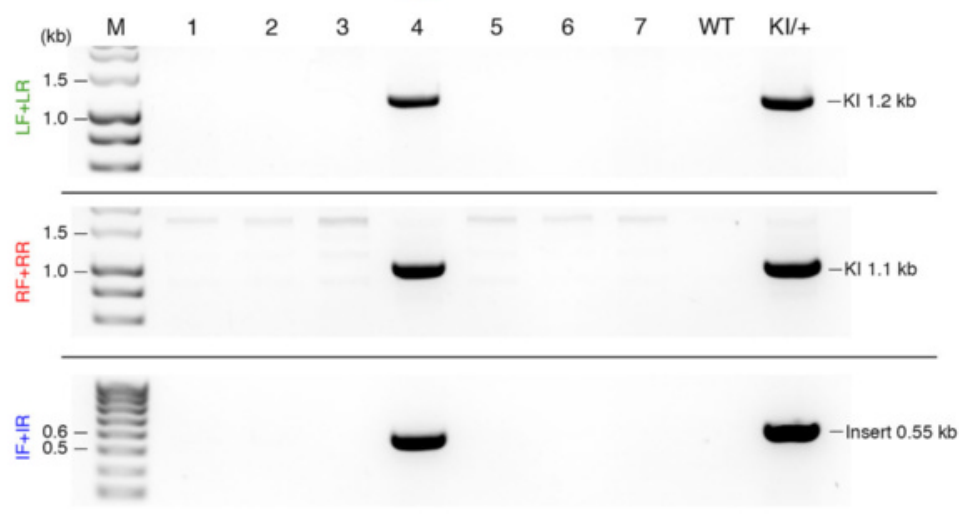

d

\begin{tabular}{cccccc} 
Method & Injected & Transferred (\%) & Newborn (\%) & Modified (\%) & Knock-in (\%) \\
\hline PITCh & 742 & $464(62.5)$ & $25(5.4)$ & $23(92.0)$ & $3(12.0)$
\end{tabular}

e

\begin{tabular}{lccc} 
5' Junction & 5' -Actb & Left-MH & TetO \\
Expected & TTCAGGAAGTGTCCAGTATCACTGTACATTGAGCATTATGAGTCCTTAAGACGCGTGTTT \\
\hline KI\#1 & TTCAGGAAGTGTCCAGTATCACTGTACATTGAGCATTATGAGTCCTTAAGACGCGTGTTT \\
KI\#2 & TTCAGGAAGTGTCCAGTATCACTGTACATTGAGCATTATGAGTCCTTAAGACGCGTGTTT \\
KI\#3 & TTCAGGAAGTGTCCAGTATCACTGTACATTGAGCATTATGAGTCCTTAAGACGCGTGTTT
\end{tabular}

3' Junction

poly A

Right-MH

3' -Actb

Expected CCCTGTCCTTTGAAGGCAAATGCATACACAAACAGGTTTGGGGCTAAGGTTCATGACCAA

KI\#1 CCCTGTCCTTTGAAGGCAAATGCATACACAAACAGGTTTGGGGCTAAGGTTCATGACCAA

KI\#2 CCCTGTCCTTTGAAGGCAAATGCATACACAAACAGGTTTGGGGCTAAGGTTCATGACCAA

$\mathrm{KI \# 3}$ CCCTGTCCTTTGAAGGCAAATGCATACACAAACAGGTTTGGGGCTAAGGTTCATGACCAA

Fig. 1 (See legend on next page.) 
(See figure on previous page.)

Fig. 1 Generation of knock-in mice carrying a gene cassette by the PITCh system. a Targeting strategy for the generation of Actb-TetO-FLEXhM3Dq/mCherry knock-in mice by the PITCh system. Purple highlights indicate microhomologies between endogenous Actb locus and PITChdonor. Blue characters indicate CRISPR target sequences. Red characters indicate protospacer adjacent motif (PAM) sequences. Yellow lightnings indicate DSB sites. $\mathbf{b}$ Schematic diagram of pronuclear injection of Cas9 protein, Actb and gRNA-s1 crRNAs, tracrRNA and PITCh-donor. The red, purple, and blue boxes indicate the insert, Actb microhomologies, and gRNA-s1 target sequences, respectively. c PCR screenings of knock-in newborns. d Summary of Actb-TetO-FLEX-hM3Dq/mCherry knock-in mouse production by the PITCh system. e Sequences of boundaries between Actb and TetO-FLEX- hM3Dq/mCherry cassette. Blue characters indicate microhomologies. IF: internal forward primer, IR: internal reverse primer, LF: left forward primer, LR: left reverse primer, RF: right forward primer, RR: right reverse primer, MH: microhomology, M: molecular marker, WT: wildtype, KI: knock-in, WPRE: woodchuck hepatitis virus posttranscriptional regulatory element, pA: polyA, and KI/+: tail genomic DNA of F1 heterozygous knock-in pup derived from \#13 (Kl\#2) F0 knock-in mouse

DNA ligase 4 [DN-Lig4], and dominant-negative Ku70 $[D N-K u 70])$ and introduced plasmids expressing these cDNAs together with three plasmids; the MMEJmonitoring reporter, CRISPR/Cas vector targeting the reporter, and mCherry-expressing vector (Fig. 2b) into HEK293T cells. Of these, we found overexpression of MMEJ-related genes and dominant-negative mutants increased the number of EGFP-positive cells (Fig. 2c, d). Especially, MMEJ efficiency by Exo1 overexpression increased more than 2.5-fold compared to that of the mock overexpression quantified by imaging analysis (Fig. 2d). The overexpression of TREX2, RAD51, or RAD52 did not increase the number of EGFP-positive cells. Conversely, significant reduction of EGFP-positive cells was observed with $R A D 52$ overexpression compared to that of mock overexpression, consistent with the involvement of this gene in the DSB repair pathway through homologous recombination [28]. These results suggest that overexpression of MMEJ factors, including Exo1, and dominantnegative NHEJ factors specifically enhanced MMEJ for DSB repair in human cells. Among these, we focused on Exo1 in this study, because Exo1 was reported to increase the mutation frequency of TALEN in mammalian cells and zygotes by potentially acting as an MMEJ enhancer [29].

\section{Exo1 enhances PITCh-mediated gene cassette knock-in in human cells}

Next, we tested whether Exo1 enhances the efficiency of PITCh-mediated gene cassette knock-in in an endogenous target locus in human cells. We constructed the PITCh-donor targeting human fibrillarin $(F B L)$ gene, a component of a nucleolar small nuclear ribonucleoprotein, containing a 1.4-kb mNeonGreen-2A-puromycin cassette $[20,21]$, although puromycin selection was not used in this study. This cassette was flanked by 40-bp left and right microhomologies corresponding to the last coding exon of the $F B L$ and gRNA-s1 crRNA target sequences (hereafter, PITCh(gRNA-s1)-FBL) (Fig. 3a). The in-frame $\mathrm{C}$-terminal fusion of $F B L$ with the cassette produces the mNeonGreen fluorescence in the nucleolar. We introduced the PITCh(gRNA-s1)-FBL donor and the all-in-one CRISPR/Cas plasmid [30] expressing two
sgRNAs targeting endogenous $F B L$ [20] and gRNA-s1, and the Cas9 protein, together with plasmids expressing Exo1 or MRE11A cDNAs in HEK293T cells (Fig. 3b). Fluorescence-activated cell sorting (FACS) analysis revealed the knock-in efficiency of the mNeonGreen cassette to the endogenous $F B L$ locus with Exo1 overexpression increased $30 \%$ compared to that with mock or MRE11A overexpression without drug selection (Fig. $3 \mathrm{c}$ and Additional file 1: Figure S4). The knock-in-enhancing effect of Exo1 was also confirmed by imaging analysis using laser-scanning microscopy (LSM). For imaging analysis, mCherry-expressing vectors were co-transfected with the above-described vectors, and the areas of mNeonGreenand mCherry-positive cells were calculated (Additional file 1: Figure S5). Consistent with the results of the FACS analysis, Exo1 increased the percentages of mNeonGreenpositive cells in mCherry-positive cells by $50 \%$ (approximately 20 vs. $30 \%$ for mock vs. Exo1 overexpression, Fig. 3d). A magnified view of the LSM images confirmed nucleolar localization of the green fluorescence foci, suggesting that the mNeonGreen-positive cells could be considered as the correctly knocked-in cells (Fig. 3e and Additional file 1: Figure S6). We further confirmed correct gene knock-in in Exo1-overexpressed cells by DNA sequencing of 5' and 3' junctions (Fig. 3f). Correct knock-in junctions without any mutations were frequently found at both 5' and 3' junctions, and their frequencies are comparable with those using a donor with 20-bp microhomology without Exo1 overexpression reported previously [21] (46.7 and 93.8\% with Exo1, 80 and 50\% without Exo1). In addition, we performed Western blotting and toxicity analysis to confirm the effectiveness and safeness of Exo1 overexpression. The results suggested that protein level of Exo1 was increased by overexpression and it did not affect cell viability (Additional file 1: Figure S7 and S8).

To confirm the knock-in-enhancing effect of Exo1, we knocked-in the same gene cassette at another gene locus in another cell line. We aimed to target human Actb (hACTB) gene in HeLa cells (Fig. 4a). The knock-in efficiency with Exo1 overexpression slightly increased compared to that without Exo1 (Fig. 4b). LSM observation revealed that fluorescent cells showed cytoplasmic localization and peripheral accumulation (Fig. 4c). The correct knock-in was 
a

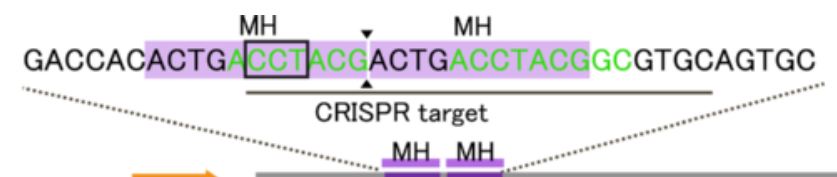

EGFP- - CMV - EGFP-N

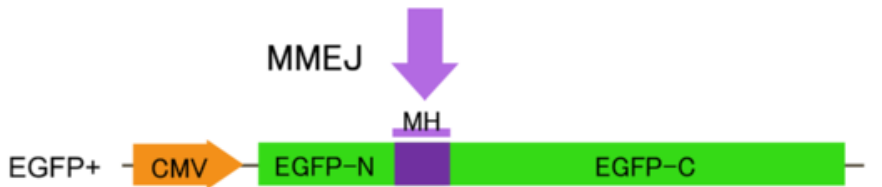

b

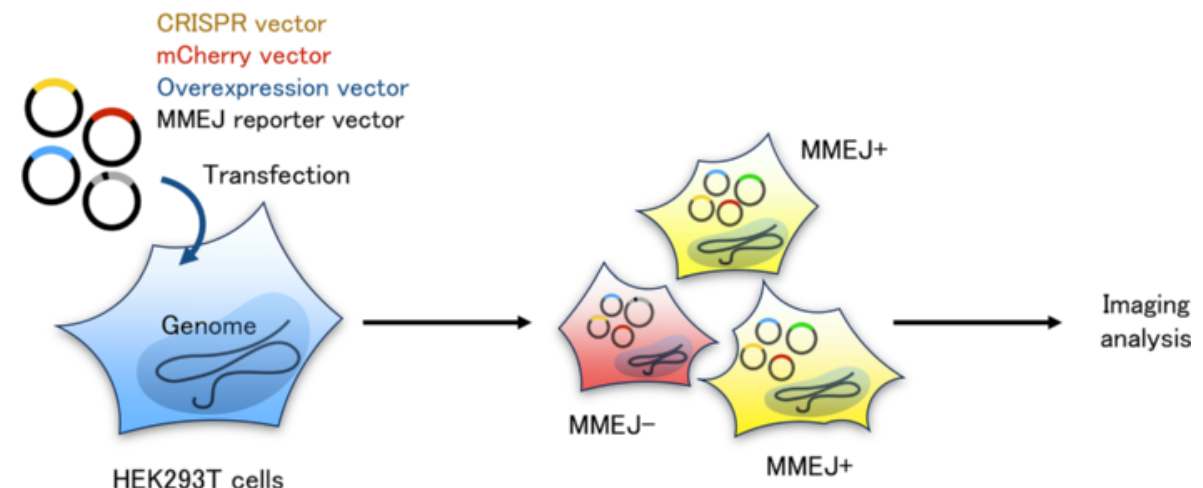

C
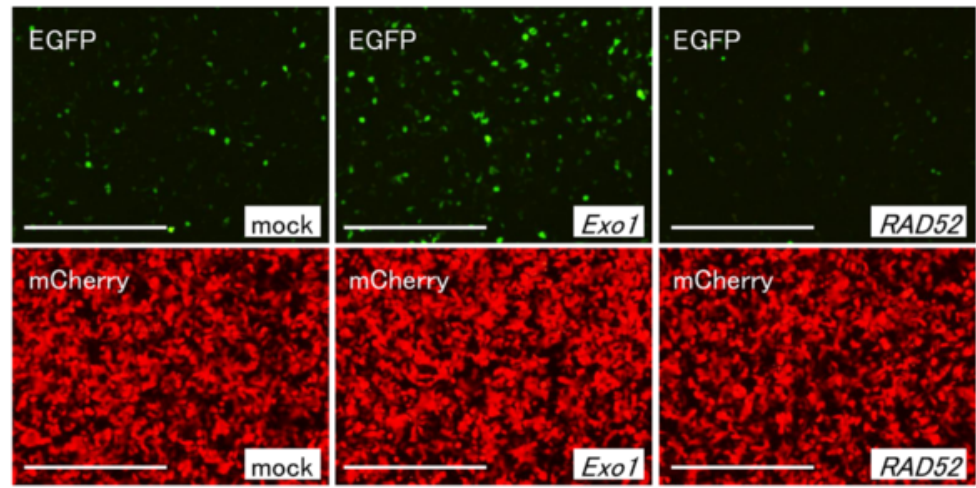

d

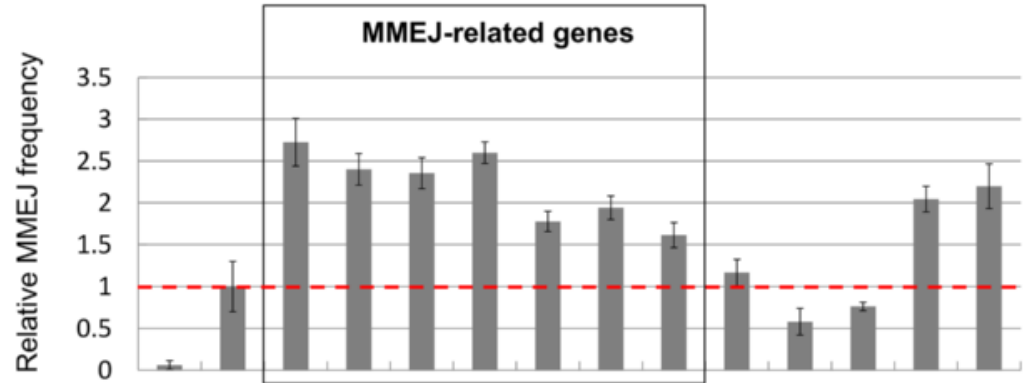

Overexpression vector

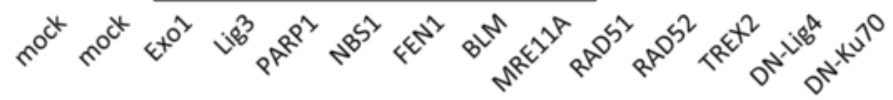

CRISPR vector Reporter vector

Fig. 2 (See legend on next page.) 
(See figure on previous page.)

Fig. 2 Screening of MMEJ-enhancing factors using a fluorescence reporter assay. a Schematic diagram of MMEJ-dependent EGFP recovery.

Green letters indicate chromophore sequence. Black line indicates CRISPR target sequence. Black box indicates protospacer adjacent motif (PAM)

sequence. Black triangles indicate DSB site. MH, microhomology. CMV, cytomegalovirus promoter. b Schematic diagram of HEK293T-based

reporter assay, followed by imaging analysis. c Fluorescence microscopy images of transfected cells. Bars, $500 \mu$ m. $\mathbf{d}$ Relative frequencies of MMEJ repair, fold to mock overexpression. The MMEJ frequencies were calculated by imaging analysis. Data are expressed as means \pm SEM $(n=4)$

also confirmed by DNA sequencing analysis (Fig. 4d, e). Without Exo1 overexpression, the percentages of precise knock-in were 47.4 and $71.4 \%$ at the 5 ' and 3 ' junctions, respectively (Fig. 4d). With Exo1 overexpression, the percentages of precise knock-in were 58.8 and $100 \%$ at the 5 ' and 3 ' junctions, respectively (Fig. 4e). From these direct comparison analysis, it was suggested that Exo1 overexpression might result in not only higher efficiency but also higher accuracy.

It has been well known that functional hACTB protein is produced from a single gene; however, there are many pseudogenes in the human genome [31]. The in silico prediction of the cleavage sites of sgRNA designed to knock-in in $h A C T B$ locus revealed that there were a lot of candidate sites in the human genome (Additional file 1: Table S1). We thus aligned the upstream and downstream regions of genomic DSB site of the on-target and 14 offtarget sites listed according to the scores calculated by the COSMID webtool [32] (Fig. 5a). Compared with the results of sequencing analysis, we found that some base replacements found in the sequenced alleles seemed to be derived from off-target integrants (highlighted in blue letters in Figs. 4d and 5a). More interestingly, mismatched bases near the DSB site were replaced with the donor sequence, whereas those far from the DSB site were not replaced, suggesting that possible window of base replacement via MMEJ repair was determined as around $15 \mathrm{bp}$ each, when the lengths of microhomologies were set as 40 bp (Fig. 5a, b). Collectively, these results suggest that Exo1 enhances the efficiency, and specificity when targeted multiple genomic sites, of the PITChmediated gene cassette knock-in in the endogenous target loci in human cells.

PITCh-mediated gene cassette knock-in in mice with Exo1 We further tested whether Exo1 enhances the efficiency of PITCh-mediated gene cassette knock-in in the endogenous target locus in mouse zygotes. We injected the circular PITCh donor with chemically synthesized Actb and $g R N A-s 1$ crRNA and tracrRNA and the Cas9 protein with in vitro-transcribed human Exo1 mRNA into one-cell stage mouse zygotes (Fig. 6a). We obtained 14 newborn mice, of these, five were identified as triple PCR positive knock-in mice carrying a TetO-FLEXhM3Dq/mCherry cassette at the Actb locus (Fig. 6b, c and Additional file 1: Figure S9). We also found one partial knock-in mouse defined by double PCR positive for
$\mathrm{LF}+\mathrm{LR}$ and IF + IR carrying a part of the cassette at the Actb locus (mouse \#2 in Fig. 6b). Next, we sequenced the PCR products of the left and right boundaries between $A c t b$ and the TetO-FLEX-hM3Dq/mCherry cassette. We found the precise knock-in of the cassette at both boundaries in four out of five knock-in mice (Fig. 6d). In eKI\#2, although the 3' boundary was precisely knocked-in as we had designed, we found a residual partial sequence of $g R N A-s 1$ adjacent to the 5' microhomology of the gene cassette (Fig. 1a) and a 57bp deletion of the endogenous locus, which did not affect endogenous $A c t b$ or exogenous cassette functions (Fig. 6d). Although left boundary was precisely knockedin in one partial knock-in mouse, we could not determine its right boundary (data not shown). The nonknock-in $A c t b$ alleles were modified by NHEJ in all the newborn mice (Fig. 6c and Additional file 1: Figure S10). Taken together, the knock-in efficiency of the gene cassette by the combination of Exo1, PITCh-donor, and cloning-free CRISPR/Cas was $35.7 \%$, with or without functionally negligible mutations (Fig. 6c). These results revealed that increasing tendency of PITCh-mediated gene cassette knock-in by Exo1 in the endogenous target locus in mouse zygotes.

We further tested off-target cleavage in the knock-in mice generated by the cloning-free CRISPR/Cas systembased PITCh system. We chose five off-target candidate loci containing up to $3 \mathrm{bp}$ mismatches compared to the 20-bp guide sequence of $A c t b$ crRNA [8]. Among five off-target candidate loci in three knock-in mice without Exo1 overexpression and five knock-in mice with Exo1 overexpression, we did not find any off-target digestion (Additional file 1: Figure S11 and Table S2). These results suggest that PITCh-mediated gene cassette knockin in mouse zygotes by the cloning-free CRISPR/Cas system is highly specific to the on-target locus, and its specificity is not affected by Exo1 overexpression.

Subsequently, we crossed F0 knock-in mice with a wild-type mouse to investigate germline transmission of the knock-in alleles to the F1 generation. We tested seven knock-in mice, and found successful germline transmission of the knock-in allele in F1 progeny (Additional file 1: Figure S12 and Table S3). The efficiencies of germline transmission were $50 \%$ or higher in five out of seven F0 knock-in mice, suggesting that these F0 mice were heterozygous knock-in mice. In contrast, two (KI\#2 and eKI\#1) showed $33.3 \%$ efficiencies of germline transmission. These 
a

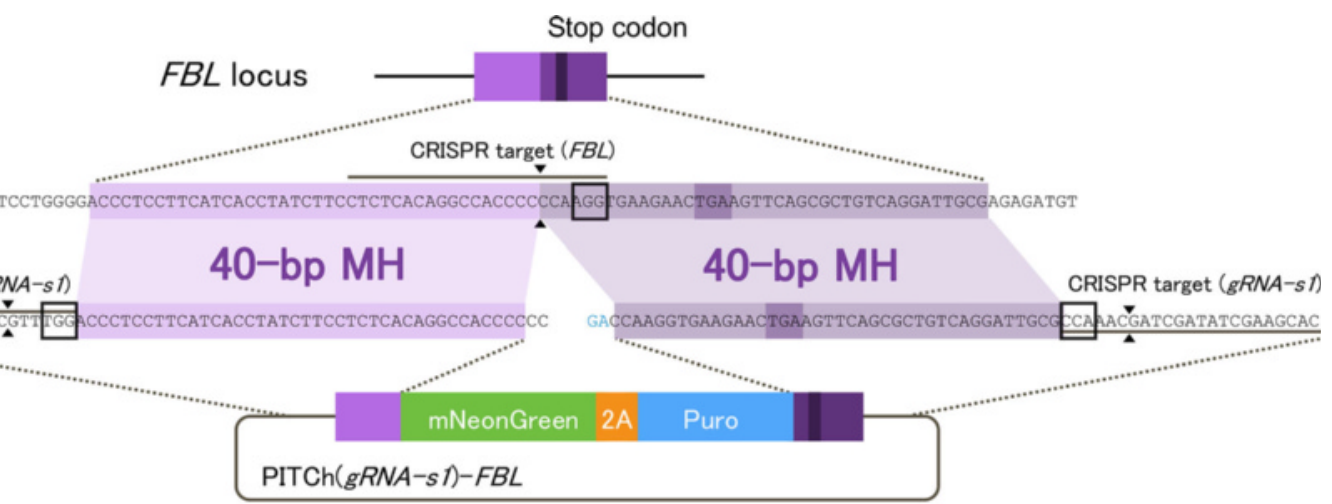

CRISPR target (gRNA-sI)

MMEJ-mediated knock-in

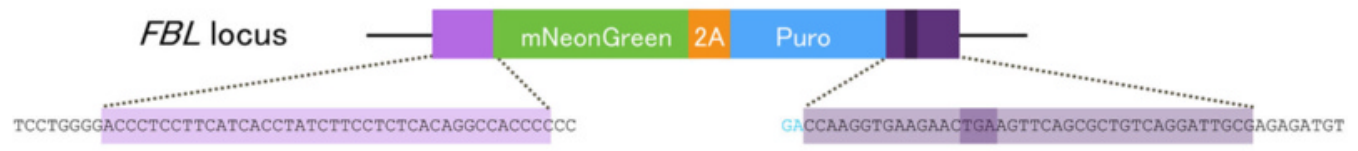

b

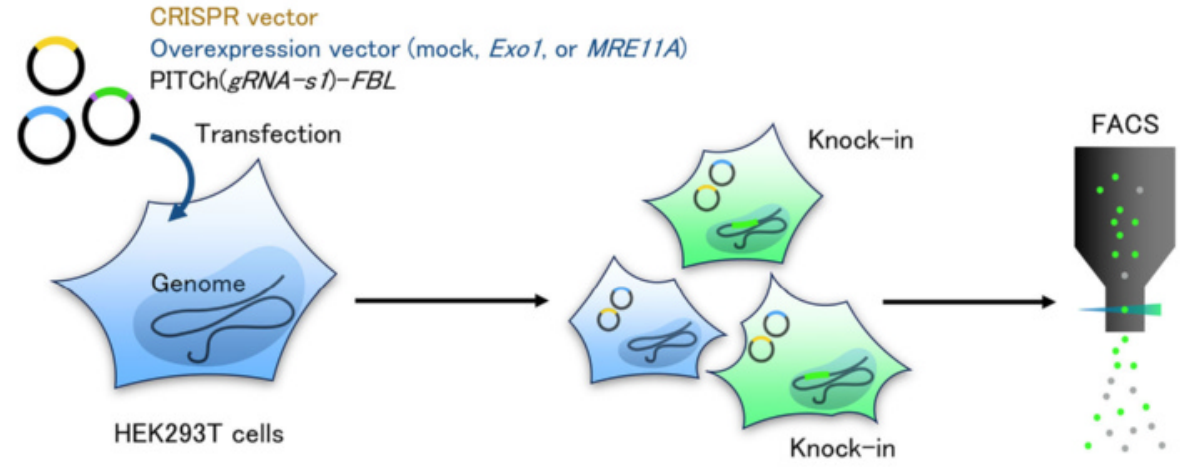

C

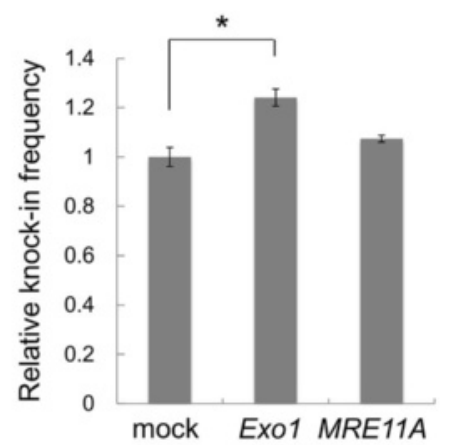

d

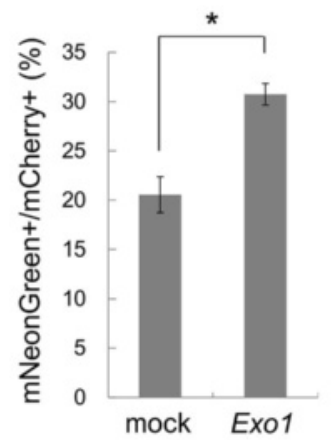

e
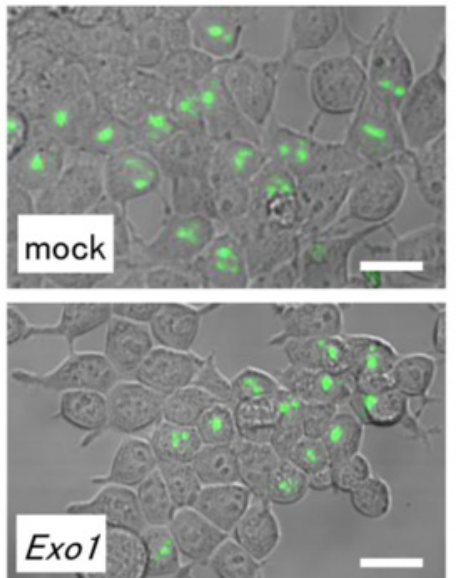

FBL locus, knock-in with Exo1, 5' junction: 7/15 (precisely knocked-in clones/total clones) CATGATCCTGGGGACCCTCCTTCATCACCTATCTTCCTCTCACAGGCCACCCCCCGGATCCATGGT

CATGATCCTGGGGACCCTCCTTCATCACCTATCTTCCTCTCACAGGCCACCCCCCGGATCCATGGT $\times 7$

CATGATCCTGGGGACCCTCCTTCATCACCTATCTTCCTCTCACAGGCCACCCCGTTTGGACCCTCCTTCATCACCTATCTTCCTCTCACAGGCCACCCCCCGGATCCATGGT X5

CATGATCCTGGGGACCCTCCTTCATCACCTATCTTCCTCTCACAGGCCACCCCGTTTGGTTCCTCTCACAGGCCACCCCCCGGATCCATGGT X1

CATGATCCTGGGGACCCTCCTTCATCACCTATCTTCCTCTCACAGGCCACCCCCCGGATTGGTTCCTCTCACAGGCCACCCCCCGGATCCATGGT X1

CATGATCCTGGGGACCCTCCTTCATCACCTATCTTCCTCTCACAGGCCACCCTCCTTCATCACCTATCTTCCTCTCACAGGCCACCCCCCGGATCCATGGT X1

FBL locus, knock-in with Exo1, 3' junction: 15/16 (precisely knocked-in clones/total clones)

GCCCGGTGCCTGACCAAGGTGAAGAACTGAAGTTCAGCGCTGTCAGGATTGCGAGAGATGTGTGTT

$\overline{\text { GCCCGGTGCCTGACCAAGGTGAAGAACTGAAGTTCAGCGCTGTCAGGATTGCGAGAGATGTGTGTT }} \times 15$

GCCCGGTGCCTGACCAAGGTGAAGAACTGAAGTTCAGCGCTGTCAGGATTGCGCCAAACCCAAGGTGAAGAACTGAAGTTCAGCGCTGTCAGGATTGCGAGAGATGTGTGTT x1

Fig. 3 (See legend on next page.) 
(See figure on previous page.)

Fig. 3 Enhancement of MMEJ-mediated gene knock-in by Exol overexpression at the FBL locus in HEK293T cells. a Schematic diagram of gene knock-in strategy at the FBL locus in HEK293T cells. Black lines indicate CRISPR target sequences. Black boxes indicate PAM sequences. Black triangles indicate DSB sites. MH, microhomology. Puro, puromycin resistance gene. $\mathbf{b}$ Schematic diagram of gene knock-in in HEK293T cells, followed by FACS analysis. c Relative frequencies of gene knock-in, fold to mock overexpression. The knock-in frequencies were calculated by FACS analysis (Additional file 1: Figure S4). Data are expressed as means \pm SEM $(n=3)$. Statistical significance was determined by Student's $t$-test. ${ }^{*} P<0.05$. $\mathbf{d}$ The percentages of mNeonGreen-positive cell areas in mCherry-positive cell areas, calculated by imaging analysis (Additional file 1: Figure S5). Data are expressed as means \pm SEM $(n=3)$. Statistical significance was determined by Student's $t$-test. ${ }^{*} P<0.05$. e Confocal laser scanning microscopy images of transfected cells. The knocked-in cells showed nucleolar localization of mNeonGreen fluorescence. Bars, $30 \mu \mathrm{m}$. $\mathbf{f}$ DNA sequencing analysis of bacterially cloned PCR products of $5^{\prime}$ and $3^{\prime}$ junctions amplified from the cells knocked-in with Exo1. The intended knocked-in sequence is shown at the top of each set of sequences. Blue letters indicate the microhomologies. Red letters indicate insertions

results suggest that germline mosaicism was occurred in some knock-in mice; however, its frequency was low.

Compared to the newborn rates of our previous work using conventional targeting vector (17\%, [8]), it was low in PITCh system (5.4\% without Exo1 and $2.5 \%$ with Exo1). We hypothesized that linearization of circular PITCh donor may partially explain this. We injected the PCR-amplified linear PITCh donor without vector backbone together with chemically synthesized $A c t b$ and $g R N A$-s 1 crRNAs and tracrRNA, and Cas9 protein into one-cell-stage mouse zygotes in the absence of Exo1 (Additional file 1: Figure S13a), and obtained only one newborn from the transfer of 191 embryos $(0.5 \%$, Additional file 1: Figure S13b). Next, we injected PCRamplified linear PITCh donor at reduced doses, Actb crRNA, tracrRNA, and Cas9 protein (Additional file 1: Figure S13a). We obtained one newborn from the transfer of 71 embryos with $5 \mathrm{ng} / \mu \mathrm{l}$ linear PITCh donor $(1.4 \%$, Additional file 1: Figure S13b). When we further reduced the dose of linear PITCh donor with $1.5 \mathrm{ng} / \mu \mathrm{l}$, the newborn rate was improved to 4.2\% (5 newborns from 119 embryo transfer) (Additional file 1: Figure S13b). These results suggest that linear donor DNA itself is detrimental to the embryo development, rather than Exo1, and the birth rate can be improved in the donor DNA dose dependent manner.

We finally tested whether modified injection protocol with reduced donor DNA concentration could produce knock-in mice by enhanced PITCh system. We chose the Col12a1 gene and designed a PITCh donor to knock-in the floxed allele for exon 2 substitution as well as a pair of crRNA targeting the flanking introns to excise the target exon (Fig. 7a) because global Col12a1knockout mice showed severe skeletal abnormalities and perinatal lethality [33]. We injected the reduced amount of circular PITCh donor $(5 \mathrm{ng} / \mu \mathrm{l})$ together with chemically synthesized Col12a1 and gRNA-s1 crRNAs, tracrRNA, Exo1 mRNA, and Cas9 protein into one-cell-stage mouse zygotes (Fig. 7b). We screened nine newborns by HindIII digestion of genomic PCR products and sequencing, and identified three as knock-in mice carrying floxed alleles (Fig. 7c-e, and Additional file 1: Figure S14). Further, we cloned and sequenced the PCR products, and found the precise knock-in of a HindIII site and two LoxP sites on the same alleles in all three knock-in mice as we designed (Fig. 7f). The precise integration of two LoxP sites on the same allele was also confirmed by excision of the allele by Cre recombinase (Fig. 7g), although Col12a1 protein level was not investigated. The knock-in efficiency of floxed Col12a1 was $33.3 \%$ (Fig. 7e) comparable to that of Actb by enhanced PITCh system (Fig. 6c). Collectively, the modified injection protocol is enough to produce the knock-in mice carrying a floxed allele by enhanced PITCh system with sufficient efficiency.

\section{Discussion}

Although we previously developed an improved CRISPR/ Cas system for HR-mediated gene cassette knock-in in mice by using chemically synthesized dual-RNAs and commercially available Cas9 protein [8], constructing targeting vectors containing long homology arms corresponding to every target locus has remained cumbersome and fundamentally unchanged for 30 years [2]. In this report, we applied the PITCh system for the generation of knock-in mice, and this resulted in the omission of the laborious steps of constructing targeting vectors. Our new method has major advantages for researchers generating gene cassette knock-in mice.

First, our method enables extremely convenient and high-throughput donor vector production. Because the MMEJ-directed donor vector can easily be prepared by a single PCR and TA-cloning using primers conjugated with 40-bp microhomology and gRNA-s1 crRNA target sequence from any template plasmid containing various gene cassettes (EGFP, Cre, and any functional gene cassettes) [21], the long homology arms, which have to be amplified from genomic DNA, are no longer required. Thus, for example, nearly $100 \mathrm{MMEJ}$-directed donor vectors corresponding to nearly 100 target loci can be prepared by a single PCR reaction in a 96-well plate and those TA-cloning, enabling a large-scale, high-throughput, knock-in mouse project $[13,14]$. By combining the enhanced PITCh system with the cloning-free CRISPR/Cas system, critical steps are to design primers to PCR amplify various gene cassettes for 40-bp microhomology arms and their subsequent cloning. Thus, our new method provides 
a

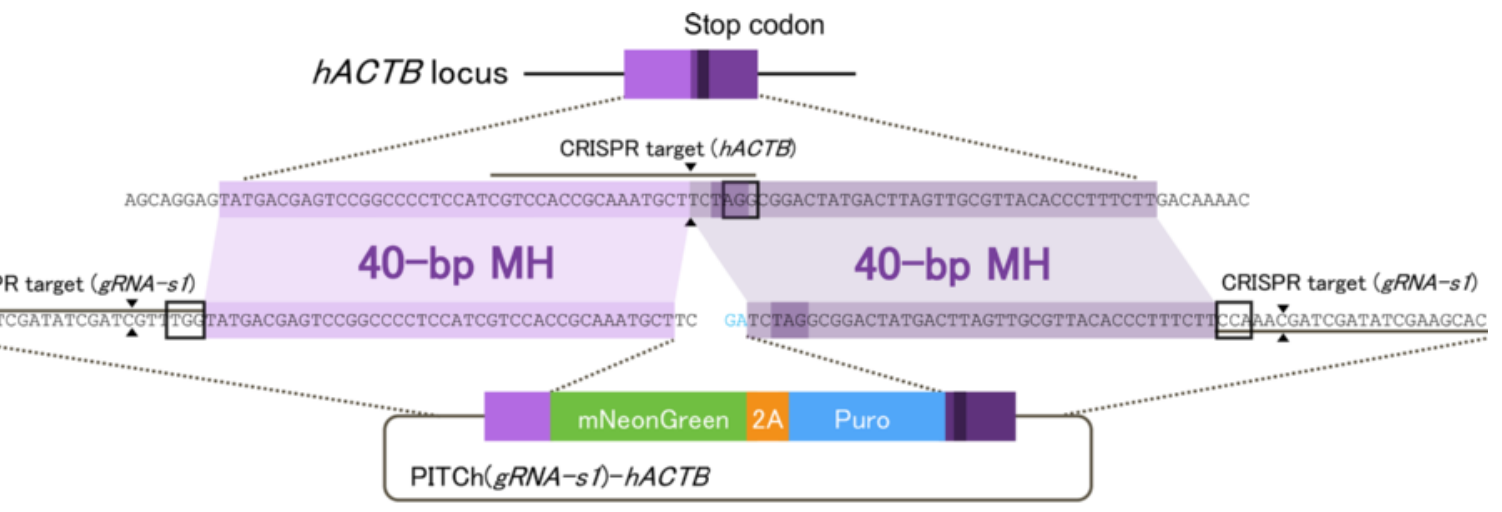

CRISPR target ( $($ RNA $-s)$

40-bp MH

40-bp MH

CRISPR target ( $(R R N A-s I)$

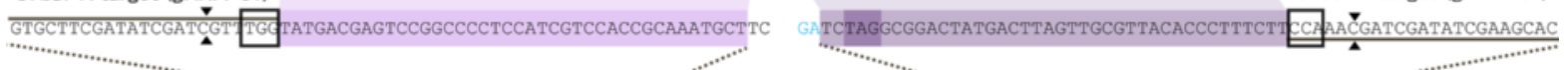

MMEJ-mediated knock-in

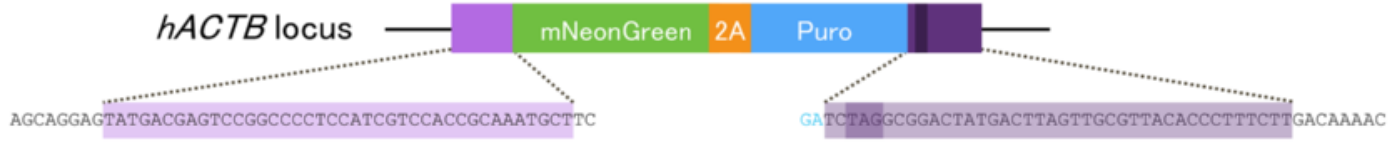

b

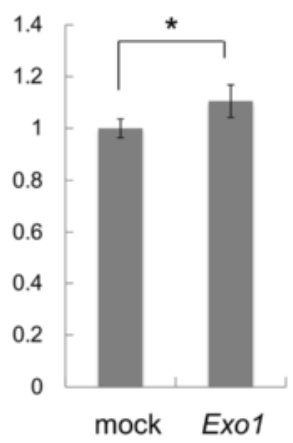

C

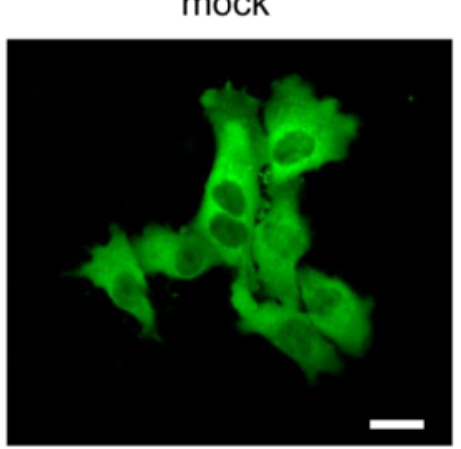

Exo1

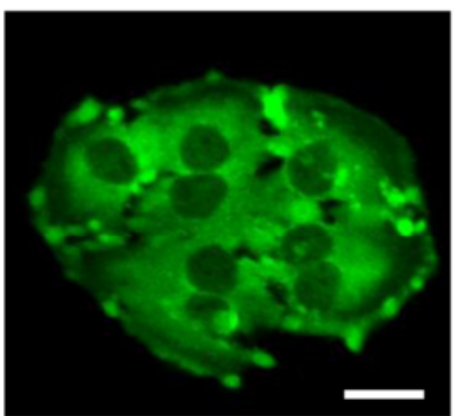

d $\quad$ dACTB locus, knock-in without Exo1, 5' junction: 9/19 (precisely knocked-in clones/total clones; 47.4\%)

CAGGAGTATGACGAGTCCGGCCCCTCCATCGTCCACCGCAAATGCTTCGGATCCATG

$\overline{\text { CAGGAGTATGACGAGTCCGGCCCCTCCATCGTCCACCGCAAATGCTTCGGATCCATG }} \times 9$

I CAGGAGTATGATGAGTCAGGCCCCTCCATTGTCCACCGCAAATGCTTCGGATCCATG $\times 3$

I I CAGGAGTATGACGAGTCCGGCCCCTCCATTGTCCACCGCAAATGCTTCGGATCCATG $\times 1$

I CAGGAGTACGATGAGTCAGGCCCCTCCATTGTCCACCGCAAATGCTTCGGATCCATG $\times 1$

CAGGAGTATGAGTCAGGACGAGTCCGGCCCCTCCATCGTCCACCGCAAATGCTTCGGATCCATG $\mathrm{x} 1$

CAGGAGTATGACGAGTCCGGCCCCTCCATCGTCCACCGCAAATGCTGTTTGGTATGACGAGTCCGGCCCCTCCATCGTCCACCGCAAATGCTTCGGATCCATG X1 CAGGAGTATGACGAGTCCGGCCCCTCCATCGTCCACCGCAAATGCTTCGTTTGGTATGACGAGTCCGGCCCCTCCATCGTCCACCGCAAATGCTTCGGATCCATG X2 CAGTTTGGTATGACGAGTCCGGCCCCTCCATCGTCCACCGCAAATGCTTCGGATCCATG x1

hACTB locus, knock-in without Exo1, 3' junction: 10/14 (precisely knocked-in clones/total clones; 71.4\%) GCCTGATCTAGGCGGACTATGACTTAGTTGCGTTACACCCTTTCTTGACAAAAC

$\overline{\text { GCCTGATCTAGGCGGACTATGACTTAGTTGCGTTACACCCTTTCTTGACAAAAC }} \times 10$

III GCCTGATCTAGGCGGACTATGACTTAGTTGCATTACACTCTTTCTTGACAAAAC $\times 2$

IV GCCTGATCTAGGCGGACTATGACTTAGTTGCATTACACCCTTTCTTGACAAAAC $\times 2$

e hACTB locus, knock-in with Exo1, 5' junction: 10/17 (precisely knocked-in clones/total clones; 58.8\%*) CAGGAGTATGACGAGTCCGGCCCCTCCATCGTCCACCGCAAATGCTTCGGATCCATG

$\overline{\text { CAGGAGTATGACGAGTCCGGCCCCTCCATCGTCCACCGCAAATGCTTCGGATCCATG }} \times 10$

CAGGAGTATGACGAGTCCGGCCCCTCCATCGTCCACCGCAAATGCTGTTTGGTATGACGAGTCCGGCCCCTCCATCGTCCACCGCAAATGCTTCGGATCCATG 33

I CAGGAGTATGATGAGTCAGGCCCCTCCATTGTCCACCGCAAATGCTTCGGATCCATG $\times 2$

CAGGAGTATGGCGAGTCCGGCCCCTCCATCGTCCACCGCAAATGCTTCGGATCCATG $\times 1$

.. (-36 bp $)$....AGTCCGGCCCCTCCATCGTCCACCGCAAATGCTTCGGATCCATG x1

hACTB locus, knock-in with Exo1, 3' junction: $17 / 17$ (precisely knocked-in clones/total clones; 100\%**)

GCCTGATCTAGGCGGACTATGACTTAGTTGCGTTACACCCTTTCTTGACAAAAC

$\overline{\text { GCCTGATCTAGGCGGACTATGACTTAGTTGCGTTACACCCTTTCTTGACAAAAC }} \times 17$

Fig. 4 (See legend on next page.) 
(See figure on previous page.)

Fig. 4 MMEJ-mediated gene knock-in at the hACTB locus in HeLa cells. a Schematic diagram of gene knock-in strategy at the hACTB locus in HeLa cells. Black lines indicate CRISPR target sequences. Black boxes indicate PAM sequences. Black triangles indicate DSB sites. MH, microhomology. Puro, puromycin resistance gene. $\mathbf{b}$ Relative frequencies of gene knock-in, fold to mock overexpression. The knock-in frequencies were calculated by FACS analysis, similar to Fig. 3b, except that mCherry was used to normalize the transfection efficiency. Data are expressed as means \pm SEM $(n=3)$. Statistical significance was determined by Student's $t$-test. ${ }^{*} P<0.05$. c Confocal laser scanning microscopy images of transfected cells. The knocked-in cells showed cytoplasmic localization and peripheral accumulation of mNeonGreen fluorescence. Bars, $30 \mu \mathrm{m}$. d, e DNA sequencing analysis of bacterially cloned PCR products of $5^{\prime}$ and $3^{\prime}$ junctions amplified from the cells knocked-in without Exo1 (d) and with Exo1 (e). The intended knocked-in sequence is shown at the top of each set of sequences. Blue bars indicate the microhomologies. Red letters indicate mutated bases including insertions, deletions, and substitutions. Blue letters indicate polymorphisms found on the off-target genomic sites, related to Fig. 5a. Roman numbers shown at the left side of each set of sequences indicate the allele types, related to Fig. 5a and Additional file 1: Figure S15, S16 and Table S1. Statistical significance was determined by chi-square test. ${ }^{*} P<0.05$. ${ }^{* *} P<0.01$

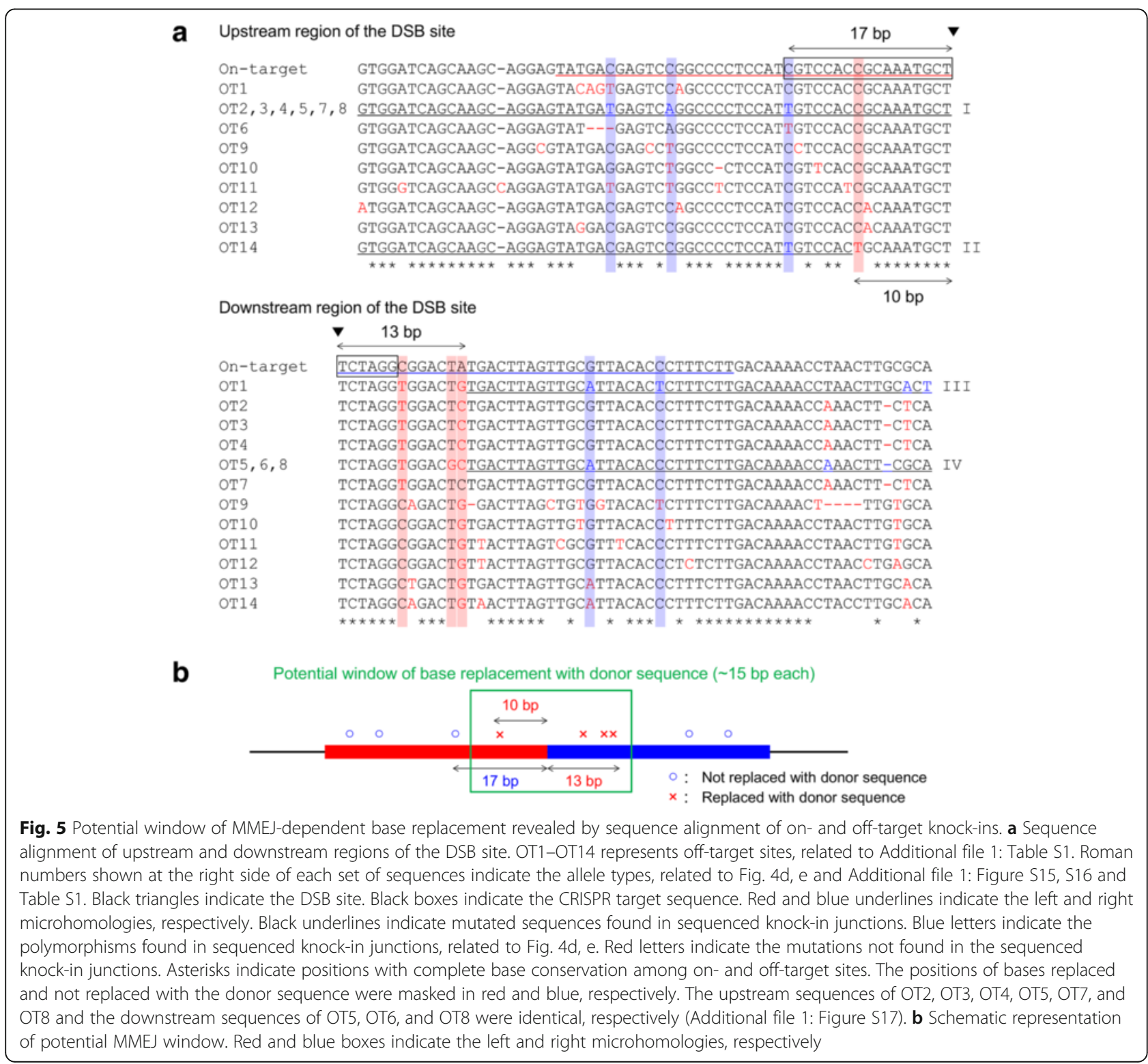




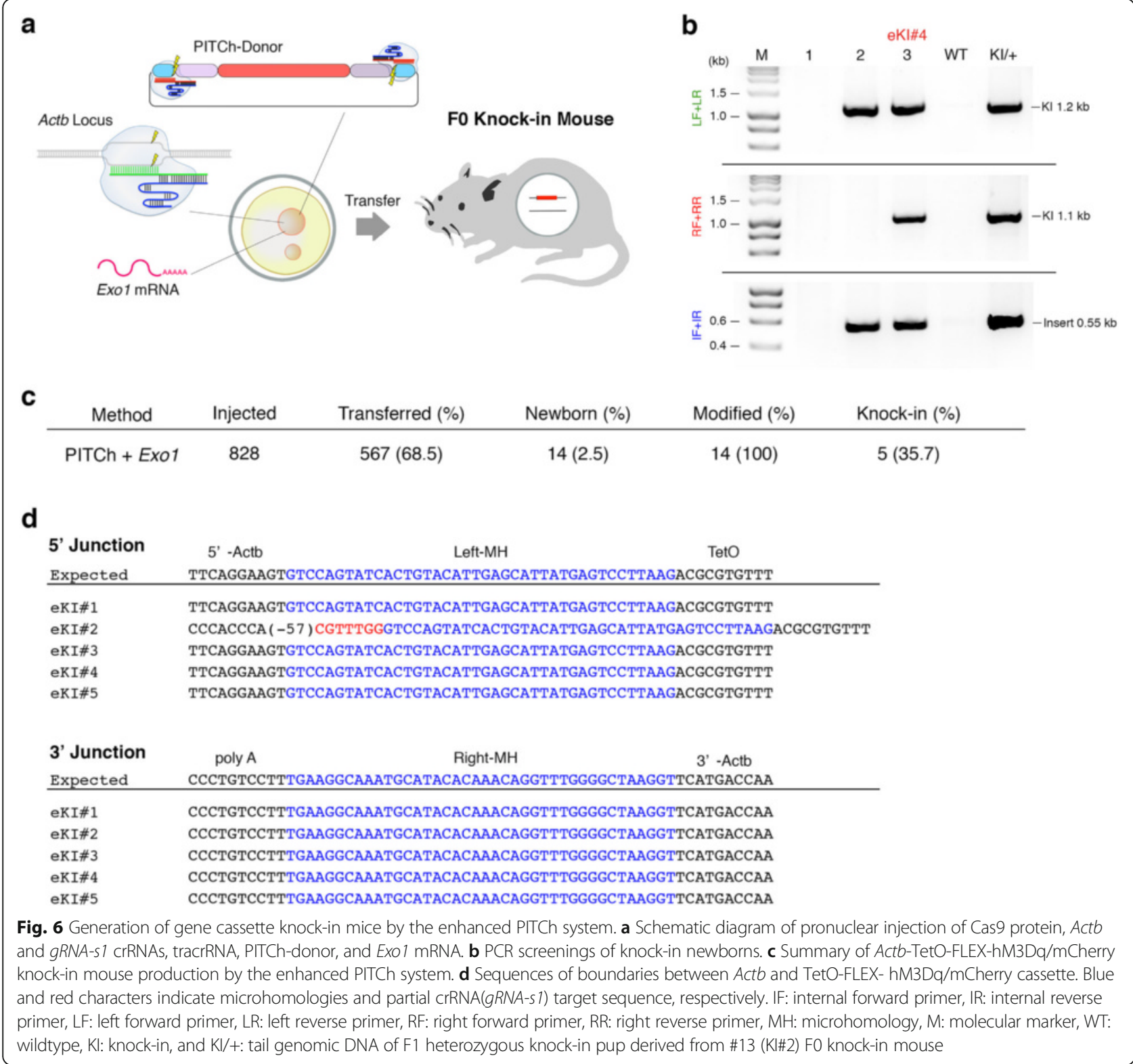

a novel approach to a knock-in gene cassette in mice on scales of both a small laboratory and a large international consortium.

Second, the enhanced PITCh system enabled gene cassette knock-in mouse generation. We successfully generated knock-in mice carrying a 5 -kb gene cassette by the original PITCh system $[20,21]$ in combination with the cloning-free CRISPR/Cas system with about $10 \%$ efficiency. Its efficiency was acceptable and comparable to previous reports using an HR-based conventional targeting vector with long homology arms $[6,10]$. We performed genetic screening to identify an MMEJ enhancer and found that the co-delivery of Exo1 enhanced knockin efficiency in human cells. We showed that the knockin efficiency of a $5-\mathrm{kb}$ gene cassette in mice was $30 \%$ by the combination of Exo1, the PITCh system, and the cloning-free CRISPR/Cas system. We further showed the validity of the enhanced PITCh system in mice in another genomic locus by knocking in floxed alleles. These efficiencies are sufficient for conventional use, and thus, several knock-in newborn mice can be obtained from small-scale embryonic manipulation, leading to flexible, high-throughput and high-variety, low-volume mouse genetics.

Despite the use of short microhomology (40 bp) in the PITCh system, the junctions between the endogenous locus and the insert were precisely integrated in the majority of the knock-in mice (21 out of 22 boundaries from 11 knock-in mice). We also found a short deletion of the endogenous locus at the $5^{\prime}$ junction in one knock- 


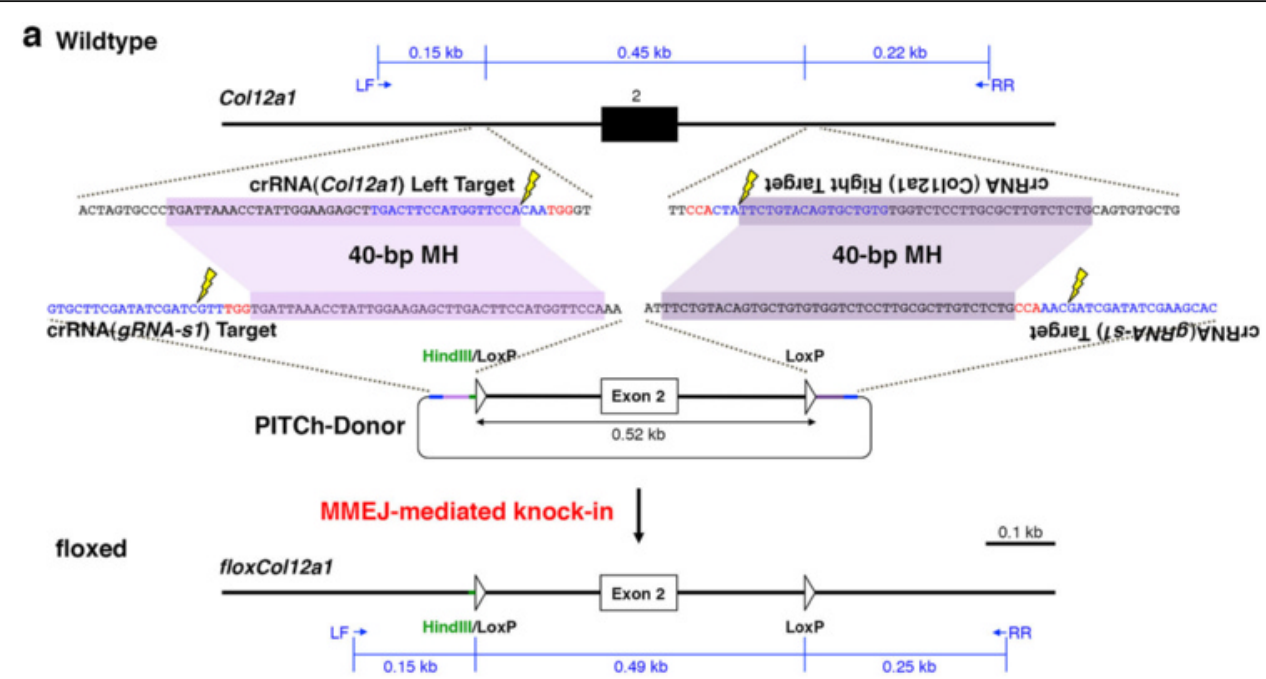

b

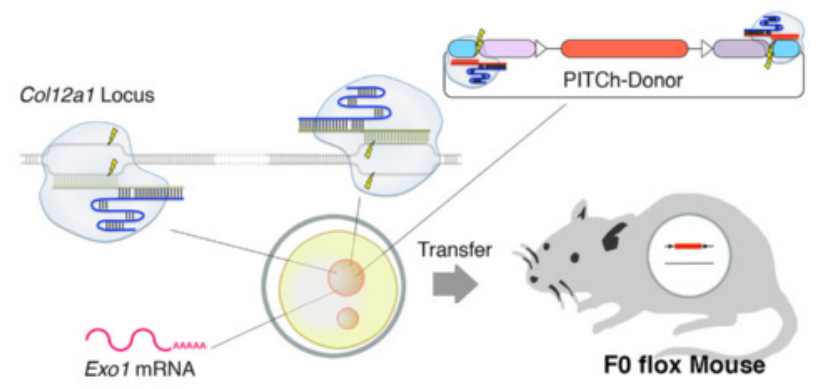

C

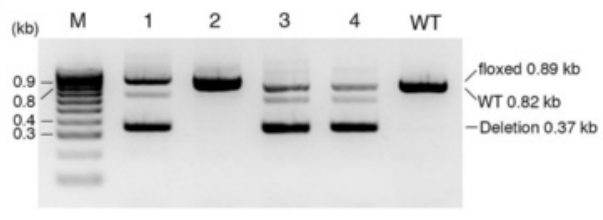

d

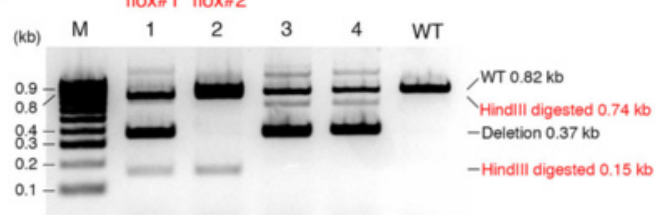

e

\begin{tabular}{cccccc} 
Method & Injected & Transferred (\%) & Newborn (\%) & Modified (\%) & floxed (\%) \\
\hline PITCh + Exo1 & 265 & $202(76.2)$ & $9(4.5)$ & $8(88.9)$ & $3(33.3)$
\end{tabular}

f
5' Junction Col12a1
Left-MH
HindIII
5' LoxP
Col12a1

Expected ACTAGTGCCCTGATTAAACCTATTGGAAGAGCTTGACTTCCATGGTTCCAAAGCTTATAACTTCGTATAGCATACATTATACGAAGTTATCAATGGGTCC

flox\#1 ACTAGTGCCCTGATTAAACCTATTGGAAGAGCTTGACTTCCATGGTTCCAAAGCTTATAACTTCGTATAGCATACATTATACGAAGTTATCAATGGGTC

flox\#2 ACTAGTGCCCTGATTAAACCTATTGGAAGAGCTTGACTTCCATGGTTCCAAAGCTTATAACTTCGTATAGCATACATTATACGAAGTTATCAATGGGTCC

flox\#3 ACTAGTGCCCTGATTAAACCTATTGGAAGAGCTTGACTTCCATGGTTCCAAAGCTTATAACTTCGTATAGCATACATTATACGAAGTTATCAATGGGTCC

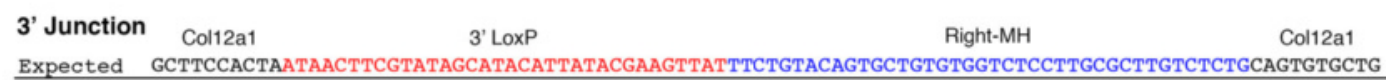

flox\#1 GCTTCCACTAATAACTTCGTATAGCATACATTATACGAAGTTATTTCTGTACAGTGCTGTGTGGTCTCCTTGCGCTTGTCTCTGCAGTGTGCTG

flox\#2 GCTTCCACTAATAACTTCGTATAGCATACATTATACGAAGTTATTTCTGTACAGTGCTGTGTGGTCTCCTTGCGCTTGTCTCTGCAGTGTGCTG

flox\#3 GCTTCCACTAATAACTTCGTATAGCATACATTATACGAAGTTATTTCTGTACAGTGCTGTGTGGTCTCCTTGCGCTTGTCTCTGCAGTGTGCTG

g

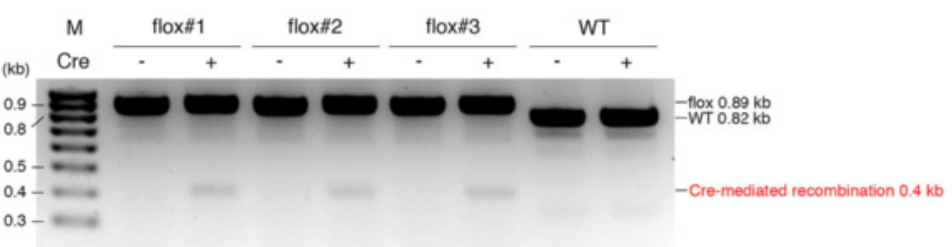

Fig. 7 (See legend on next page.) 


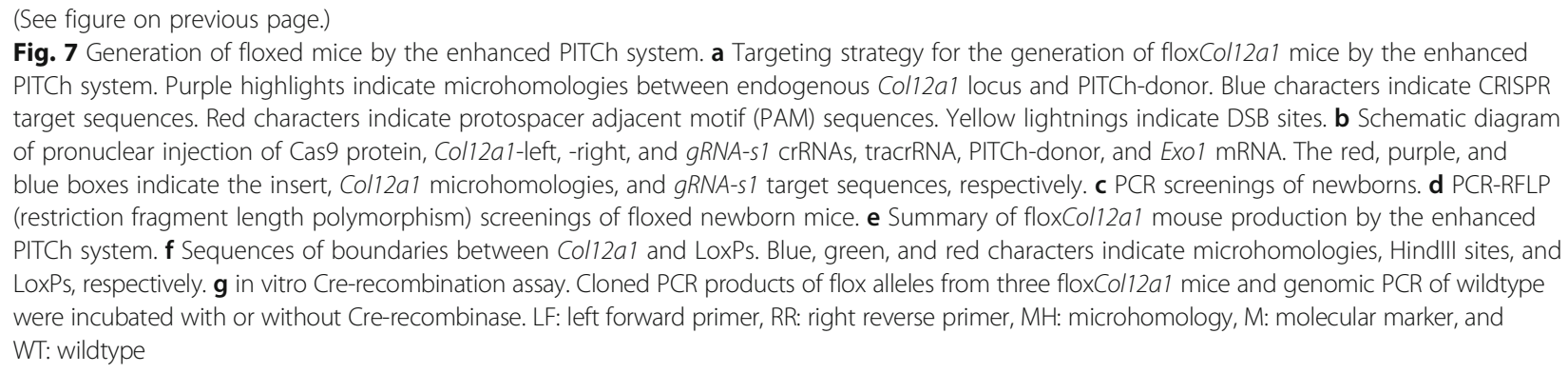

in mouse treated with Exo1, suggesting NHEJ-mediated repair occurred between the deleted genome and the digested donor end. Although the imprecise repair around the targeted integration locus did not affect endogenous gene or exogenous cassette functions when the introns or intergenic regions were targeted, further technological improvements are required for precise targeting of the coding regions to fuse the gene cassettes with endogenous gene products. In addition, we found a few partial knock-in mice defined by double PCR positive for LF + LR and IF + IR (e.g. mice \#10 and \#18 in Additional file 1: Figures S2 and mouse \#2 in Fig. 6b) in the presence or absence of Exo1. The whole plasmid integration by insufficient digestion of right boundary of the PITCh-donor or deletion of primer-binding site in mouse genome or PITCh-donor caused by DNA end resection might result in failure of right boundary amplification. Nevertheless, the enhanced PITCh system can generate knock-in mice with sufficient efficiency, and the majority of these mice had precise integration of the gene cassette to the endogenous locus, providing opportunities to select precisely targeted knock-in mice.

We found Exo1 as an MMEJ enhancer both in human cells and mouse zygotes. Exo1 is a major 5'-3' exonuclease, which is critical for the end resection of DSBs to promote MMEJ [17]. The mutation and overexpression of Exo1 in yeasts are reported to reduce and increase MMEJ, respectively [34, 35]. Consistent with this view, we recently reported that the overexpression of Exo1 increased knockout efficiency possibly through increased MMEJ in rat zygotes [29]. Thus, in the enhanced PITCh system, Exo1 may act as the MMEJ enhancer by resection of the DSB ends of both the endogenous locus and the donor vector, promoting their alignment using microhomology. We also showed that various MMEJrelated genes and dominant-negative mutants of NHEJ factors enabled to enhance MMEJ. The enhancer effect of these genes in gene knock-in should be examined in the future study. On the other hand, additional three genes (TREX2, RAD51, and RAD52) involved in the repair pathway of DSBs did not enhance MMEJ. TREX2 is also a DNA end-processing enzyme, which reportedly enhances gene disruption when overexpressed with site- specific nucleases [36]. However, it slightly decreased MMEJ frequency, suggesting that it is involved in other DSB repair pathways rather than in the MMEJ pathway. Similarly, RAD51 and RAD52, known as HR-specific factors [28], also decreased the MMEJ frequency.

We additionally investigated whether mismatched bases in microhomologies can be replaced with the donor sequence during the PITCh knock-in, by simultaneously targeting the $h A C T B$ locus and other pseudogenes. Although there might be an amplification bias of 5 ' and 3 ' junctions depending on the sequence variations between primers and target sites (Additional file 1: Figure S15), we found two types of off-target knock-in alleles in 5 ' junction and another two types in 3 ' junction. The sequence comparison between genomic sequences and sequenced alleles revealed that the base-replacing window was around $15 \mathrm{bp}$. This parameter might be changed when the different length of microhomology is used or different site-specific nucleases such as TALENs are used, but this finding would be important to design both the target site and microhomologies for PITCh knock-in, especially when multiple genomic loci are simultaneously targeted.

The injection of linear PCR donors was enough to reduce the newborn mouse rates in a dose dependent manner. Consistent with our data, Miura et al. also reported that injection of linear double strand DNA donor strongly reduce the newborn mouse rates [37]. These results suggest that the low birth rate of newborn mice in PITCh system is mainly caused by toxicity of linear donor DNA generated by CRISPR-mediated in situ digestion. The survival rate can be improved by the reduction of PITCh donor dose. We showed this modified protocol could also produce knock-in mice without reduction of its efficiency. Although transfection of the various doses of Exo1 expressing plasmids to HEK293T cell did not affected their survival, its overexpression in mouse zygotes reduced the newborn rate. A recent report in the human cell line showed that overexpression of TREX2, an end-processing enzyme, increased both on- and off-target mutation rates [38], whereas other showed opposite results [36]. This suggests the possibility that the overexpression of Exo1 may also increase 
off-target mutation rate, leading to the reduction of newborn mouse rate, and thus, the careful guide RNA design is also essential.

The development of small molecules that activate Exo1 may be useful for improvement of knock-in efficiency. Scr7, a small compound inhibitor of DNA ligase IV, which is responsible for NHEJ, was recently developed [39] and harnessed to improve the efficiency of HR-mediated knock-in of single nucleotide substitutions and small tags in mice [12, 40] and human cells [41] by suppressing NHEJ. Further, the two small compounds (L755507 and Brefeldin A) were also identified as enhancers for HR by chemical screening using the fluorescent HR reporter system [42]. Similarly, RS-1, a stimulator of Rad51 [43], is shown to enhance knock-in of long inserts in cellular knock-in screening [44] and rabbits [45]. Thus, by utilizing the fluorescent reporter system to detect MMEJ-mediated repair of DSBs, which we used herein, the small compounds that enhance MMEJ and PITCh-mediated gene cassette knock-in could be identified.

\section{Conclusions}

We developed a convenient HR-independent method for one-step generation of knock-in human cells and mice carrying gene cassettes and floxed allele without long homology arms by enhanced PITCh system.

\section{Methods}

\section{Plasmid construction for human cell experiments}

The CRISPR/Cas9 vector expressing Cas9 nuclease and an sgRNA targeting EGFP reporter vector was constructed using pX330 vector (Addgene Plasmid \#42230) according to the previously described protocol [46]. The all-in-one CRISPR/Cas9 vector expressing Cas9 nuclease and two sgRNAs targeting the $F B L$ or $h A C T B$ gene and the donor vector was constructed using the Multiplex CRISPR/Cas9 Assembly System Kit (Addgene Kit \#1000000055) as described previously [30]. The oligonucleotide sequences for sgRNA templates were listed in Additional file 1: Table S4.

The overexpression vectors were constructed using an RT-PCR and In-Fusion HD Cloning Kit. Briefly, the full coding sequences for the 14 genes listed in Fig. $2 \mathrm{~d}$ were amplified using an RT-PCR from total RNAs extracted from HEK293T cells. The amplified cDNAs were cloned into ptCMV-136/63-VR-NG vector (Addgene Plasmid \#50700) [47], replacing the transcription activatorlike effector nuclease-coding sequence with each cDNA. The sequences of primers used to construct the overexpression vectors were listed in Additional file 1: Table S4.

The EGFP reporter vector for monitoring MMEJ frequency was constructed using a PCR and In-Fusion HD Cloning Kit (Takara). PITCh(gRNA-s1)-FBL and $\operatorname{PITCh}(g R N A-s 1)-h A C T B$ donor plasmids were constructed using a PCR and TA-cloning with DynaExpress TA PCR Cloning Kit (pTAC-2) (BioDynamics Laboratory Inc.) or TArget Clone -Plus- (Toyobo). The full plasmid sequences of EGFP reporter vector, $\operatorname{PITCh}(g R N A-s 1)-F B L$ donor vector, and PITCh(gRNA-s1)-hACTB donor vector are shown in Additional file 1: Figure S17.

\section{Cell culture and transfection}

HEK293T and HeLa cells were maintained in Dulbecco's modified Eagle's medium supplemented with $10 \%$ fetal bovine serum. Lipofectamine LTX (Life Technologies) and Opti-MEM (Life Technologies) were used to transfect plasmids, according to the manufacturer's instructions. Plasmid concentrations, cell numbers, and dishes used were as follows: for the MMEJ-monitoring reporter assay, $100 \mathrm{ng}$ each for all the plasmid vectors (EGFP reporter vector, CRISPR/Cas vector, mCherry vector, and each overexpression vector) into $6 \times 10^{4}$ HEK293T cells using a 96-well plate; for the FACS and DNA sequencing analyses and the imaging quantification using LSM of $F B L$ knocked-in cells, $100 \mathrm{ng}$ each for all the plasmid vectors (PITCh donor vector, CRISPR/Cas vector, and mock, Exo1, or MRE11A vector with or without mCherry vector) into $5.0 \times 10^{4} \mathrm{HEK} 293 \mathrm{~T}$ cells using a 12-well plate; for the fluorescence observation of $F B L$ knocked-in cells, $50 \mathrm{ng}$ each for all the plasmid vectors (PITCh donor vector, CRISPR/Cas vector, mock or Exo1 vector, and mCherry vector) into $3 \times 10^{4}$ HEK293T cells using a 96-well plate; for gene knock-in at the $F B L$ locus and co-transfection of FBL-mCherry fusion gene, $100 \mathrm{ng}$ each for all the plasmid vectors (PITCh donor vector, CRISPR/Cas vector, and $F B L$-mCherry vector) into $2.5 \times 10^{4}$ HEK293T cells using a 12-well plate; for the FACS analysis of $h A C T B$ knocked-in cells, $100 \mathrm{ng}$ each for all the plasmid vectors (PITCh donor vector, CRISPR/Cas vector, mCherry vector, mock or Exo1 vector, and the vector expressing puromycin resistance gene) into $2 \times 10^{5} \mathrm{HeLa}$ cells using a 12-well plate; for the DNA sequencing and fluorescence observation of $h A C T B$ knocked-in cells, 8.3-50 ng each for all the plasmid vectors (PITCh donor vector, CRISPR/Cas vector, mCherry vector, mock or Exo1 vector) into $3 \times 10^{4} \mathrm{HeLa}$ cells using a 96-well plate; for Western blotting analysis, $2 \mu \mathrm{g}$ for mock or Exo1 vector into $5 \times 10^{4}$ HEK293T cells using a 6 -well plate; for toxicity analysis, $1,3.5,12.2,42.9$, and $150 \mathrm{ng}$ each for mock or Exo1 vectors, or $150 \mathrm{ng}$ for zinc-finger nuclease (ZFN) vector, pSTL-ZFA36 [48], and $150 \mathrm{ng}$ for mCherry vector into $6 \times 10^{4}$ HEK293T cells using a 96-well plate. After transfection, cells were cultured in the growth medium described above for 1 day (reporter assays), 3 days (knock-in experiments and Western blotting), or 2 or 5 days (toxicity analysis), with or without transferring the cells to a larger culture plate. For the FACS analysis of $h A C T B$ knocked-in cells, puromycin selection was conducted during 24-72 h post-transfection. 


\section{FACS analysis}

Cells were collected, suspended in PBS, and filtered with a Flowmi Tip Strainer (Bel-Art Products). The number of cells with green (mNeonGreen) and red (mCherry) fluorescence, where needed, was counted using a BD FACS Calibur 4A (BD Biosciences) with a 488-nm laser and the corresponding fluorescence filters. A total of 10,000 cells were recorded for each sample. For toxicity analysis, cell survival rates were determined as the percentages of the number of mCherry-positive cells in total cell counts, as described previously [49].

\section{Fluorescence microscopy}

For the reporter assays, fluorescence was observed and cell images were captured using a fluorescence microscope (Olympus CKX41) directly in the cultured plates. For the knock-in experiments, cells were moved to collagen-coated glass-bottom 24-well plates at $72 \mathrm{~h}$ post-transfection, cultured for additional $24 \mathrm{~h}$, and fixed with $4 \%$ paraformaldehyde in PBS. Fluorescence was observed and cell images were captured with a 473-nm and 594-nm lasers using a confocal laser-scanning microscope (Olympus FV-1000D).

\section{Imaging analysis using the ImageJ software}

For the reporter assays, the areas containing red fluorescence (mCherry) and green fluorescence (EGFP) were calculated from the captured images using the ImageJ software (https://imagej.nih.gov/ij/). The MMEJ efficiency was determined as the percentages of the EGFP-positive areas in the mCherry-positive areas. For the knock-in experiments, imaging analysis was performed as described in Additional file 1: Figure S4.

\section{Sequencing analysis for human cell experiments}

The 5' and 3' knock-in junctions of $F B L$ and $h A C T B$ genes were amplified by PCR using the primers listed in Additional file 1: Table S4 from the cells collected at $72 \mathrm{~h}$ post-transfection without any antibiotic or fluorescence selection. Subsequently, the PCR products were bacterially cloned using the TA-cloning method and sequenced.

\section{Western blotting}

Cells were collected and dissolved in sample buffer (final concentrations: $0.125 \mathrm{M}$ Tris- $\mathrm{HCl}, \mathrm{pH} 6.8$, 4\% SDS, $20 \%$ glycerol, $10 \% \beta$-mercaptoethanol, $0.005 \%$ bromophenol blue). Western blotting was performed as previously described [50]. Briefly, after denaturation by heating at 95 degree for $10 \mathrm{~min}, 10 \mu \mathrm{g}$ of each sample was separated by 4-20\% SDS-PAGE (Bio-Rad) along with a protein standard ladder (Nippon Genetics). The samples were then transferred to PVDF membranes. The membranes were cut and blocked in TBS with $0.1 \%$ Tween 20 (TBS$\mathrm{T}$ ) and $5 \%$ skim milk for $1 \mathrm{~h}$ at room temperature, then incubated with primary monoclonal antibodies against Exo1 (1:100, Thermo Scientific, Ab-4, clone 266) and $\beta$ Actin (1:1000, Santa Cruz Biotechnology, sc-47778) in TBS-T containing $1 \%$ skim milk at $4^{\circ}$ overnight. Next, the membranes were washed, incubated with HRP-conjugated anti-mouse IgG secondary antibody (1:5000, Jackson ImmunoResearch Laboratories, 715-035-151) at room temperature for $1 \mathrm{~h}$, washed, and visualized with Luminata Forte Western HRP substrate (Millipore). Gel images were taken every $1 \mathrm{~min}$ for $20 \mathrm{~min}$ using Image Lab software (Bio-Rad). Band intensities of gel images within linear signal range were quantified using Image Lab software and normalized with band intensities of $\beta$-Actin.

\section{Animal experiments}

All research and animal care procedures were approved by the Tokyo Medical and Dental University Animal Care and Use Committee. Mice were housed in groups of 3-5 animals per cage and maintained on a regular $12 \mathrm{~h}$ light/dark cycle (8:00-20:00 light period) at a constant $25{ }^{\circ} \mathrm{C}$. Food and water were available ad libitum.

\section{Targeting vector for mouse experiments} Actb-TetO-FLEX-hM3Dq/mCherry PITCh-donor: TetOFLEX-hM3Dq/mCherry cassette was PCR-amplified from an original plasmid with PrimeSTAR GXL DNA Polymerase (Takara) and primers conjugated with $A c t b$ microhomologies and gRNA-s1 crRNA target sequences (Fig. 1a), or with $A c t b$ microhomologies (Additional file 1: Figure S13). PCR products were directly used as linear PITCh donors (Additional file 1: Figure S13). Then, PCR products were cloned into plasmids using Mighty TAcloning Kit (Takara). The PCR products were also inserted into plasmids using In-Fusion HD Cloning Kit (Takara), in some cases.

floxCol12a1 PITCh-donor: The genomic region containing exon 2 of Col12a1 was PCR-amplified as described above with primers conjugated with LoxPs. Then, second PCR was performed with primers conjugated with Col12a1 microhomologies and gRNA-s1 crRNA target sequences. Then, PCR products were cloned into plasmids using Mighty TA-cloning Kit (Takara).

\section{in vitro mRNA transcription}

Exo1 mRNA was in vitro transcribed using mMESSAGE mMACHINE T7 ULTRA Kit (Life Technologies). Exo1 mRNA was purified with MEGAclear Kit (Life Technologies) and eluted with Nuclease-free water (Life Technologies). The quality of mRNA was analyzed by NanoDrop (Thermo Scientific) and Bioanalyzer (Agilent Technologies).

\section{Cas9 proteins}

The recombinant Cas9 proteins were obtained from Fasmac, New England BioLabs (NEB), and PNA Bio. 


\section{Chemical synthesis of crRNA and tracrRNA}

crRNAs and tracrRNA (Additional file 1: Table S4) were chemically synthesized and purified by high pressure liquid chromatography (Fasmac).

\section{in vitro digestion assay}

IDAs were performed as previously described [8] with $100 \mathrm{ng}$ of the PITCh-donor vector, gRNA-s1 crRNA, tracrRNA and the Cas9 protein.

\section{Injection}

Injections were performed as previously described [8]. For Actb-TetO-FLEX-hM3Dq/mCherry knock-in mice, Cas9 proteins, Actb and gRNA-s1 crRNAs, tracrRNA, and PITCh-donor vectors with or without Exo1 mRNA (30 ng/ $\mu \mathrm{l}, 0.61 \mathrm{pmol} / \mu \mathrm{l}, 10 \mathrm{ng} / \mu \mathrm{l}$, and $10 \mathrm{ng} / \mu \mathrm{l}$, respectively) were injected into pronuclei of one-cell stage BDF1 zygotes. For linear PCR donor injection, Cas9 proteins, Actb crRNA (and gRNA-s1 crRNA), tracrRNA, and linear PCR PITCh-donor $(1.5,5$, or $10 \mathrm{ng} / \mu \mathrm{l})$ were injected. For floxCol12a1 mice, Cas9 proteins, left and right Col12a1 crRNAs, tracrRNA, and PITCh-donor vectors with Exo1 mRNA $(30 \mathrm{ng} / \mu \mathrm{l}, 0.61 \mathrm{pmol} / \mu \mathrm{l}$, $5 \mathrm{ng} / \mu \mathrm{l}$, and $10 \mathrm{ng} / \mu \mathrm{l}$, respectively) were injected into pronuclei of one-cell stage C57BL/6 J zygotes. After incubation at $37^{\circ}$, one-cell stage embryos were transferred into pseudopregnant ICR female mice.

\section{PCR screening}

PCR screenings of knock-in mice were performed with primers listed in Additional file 1: Table S4 as previously described [8]. PCR products were directly sequenced or cloned, then sequenced. For floxCol12a1 screening, PCR products were digested with HindIII (NEB).

\section{in vitro Cre-recombination}

$200 \mathrm{ng}$ of PCR products of cloned floxCol12a1 alleles from floxed mice or genomic Col12a1 PCR products of wildtype mice were incubated with Cre recombinase (NEB) and analyzed by $2 \%$ agarose gel electrophoresis according to the manufacture's instruction.

\section{Off-target effects of knock-in mice}

The potential off-target candidate loci were analyzed as previously described [8]. PCR primers were listed in Additional file 1: Table S4.

\section{Statistical analyses}

All data are presented as the mean \pm SEM. Statistical methods were described in the figure legends for each data set. Statistical significance was set at $p<0.05$.

\section{Additional file}

\begin{abstract}
Additional file 1: The following additional data are available with the online version of this paper. Additional data file 1 contains the figures including IDA analysis of PITCh-donor, PCR screenings of mice, sequencing of non-knock-in Actb alleles, FACS and LSM analysis of human cells, Exo1 western blotting, Exo 1 toxicity analysis, off-target analysis in mice, germline transmission, linear PCR donor injection, and sequence alignments of $h A C T B$ and its off-target sites, and tables of these results and a list of the oligo DNAs and RNAs used in this study. (DOCX $14447 \mathrm{~kb}$ )
\end{abstract}

\section{Abbreviations}

CRISPR/Cas: Clustered regularly interspaced short palindromic repeat (CRISPR)/CRISPR-associated protein (Cas); crRNA: Crispr RNA;

DREADD: Designer receptors exclusively activated by designer drug; DSBs: Double-strand breaks; EGFP: Enhanced green fluorescent protein; Exo 1: Exonuclease 1; FACS: Fluorescence-activated cell sorting; FBL: Fibrillarin; HR: Homologous recombination; IDA: In vitro digestion assay; MMEJ: Microhomology-mediated end joining; NHEJ: Nonhomologous end joining; PA: PolyA; PAM: Protospacer adjacent motif; PITCh: Precise integration into the target chromosome; sgRNA: Single-guide RNA; tetO: TetO operator; tracrRNA: Trans-activating crRNA; WPRE: Woodchuck hepatitis virus posttranscriptional regulatory element

\section{Acknowledgements}

We thank the genome editing facility at laboratory of recombinant animals, MRI, TMDU, K. Watase (CBIR, TMDU), K.F. Tanaka (Keio Univ.), A. Yamanaka (Nagoya Univ.), and Y. Wada (Fasmac) for providing materials and useful discussions, and A. Kawahara (Yamanashi Univ.) and Y. Hisano (RIKEN) for co-developing the CRISPR/Cas-mediated PITCh system. We also thank Y. Hara (Hiroshima Univ.) for technical assistance, and H. Ochiai (Hiroshima Univ.) for sharing the synthesized mNeonGreen cDNA under the license agreement with Allele Biotechnology and Pharmaceuticals, Inc [51].

\section{Funding}

This work was supported by the Grant-in-Aid for Young Scientists (B) (26830131) and the Grant-in-Aid for Scientific Research (C) (16 K07085) to T.A., the Grant-inAid for Young Scientists (B) (15 K19988) to Y.I., and the Grant-in-Aid for Young Scientists (B) (16 K18478) to T.S. from Japan Society for the Promotion of Science, the Grant-in-Aids for Scientific Research on Innovative Areas (Comprehensive Brain Science Network) to T.A. and (Adaptive Circuit Shift) to H.A. and T.A.(26112010), and the Strategic Research Program for Brain Science to K.T. from The Ministry of Education, Culture, Sports, Science and Technology of Japan, and grants to T.A. from Nakatani Foundation, Takeda Science Foundation, SENSHIN Medical Research Foundation, Mochida Memorial Foundation for Medical and Pharmaceutical Research, MRI of TMDU, and the president of TMDU, and the joint Usage/Research Program from MRI of TMDU, and grant BS281001 from Brain Sciences Project of the Center for Novel Science Initiatives (CNSI), National Institutes of Natural Sciences (NINS) to K.T. and T.A., and grants to Y.I. from the Nakatomi Foundation, the Ichiro, Kanehara Foundation, and Takeda Science Foundation. This work was also supported by grants from the Sasakawa Foundation to S.N., the Uehara Memorial Foundation to T.S., and the Ministry of Health, Labor, and Welfare of Japan to T.Y. The funders have no role in the design of the study and collection, analysis, and interpretation of data and in writing the manuscript should be declared.

\section{Availability of data and materials}

All the data supporting findings in this manuscript is contained within the manuscript.

\section{Authors' contributions}

TA and TS conceived the study. TA, TS, and KT wrote the manuscript. TA, TS, and SN designed all the experiments and analyzed the data. TA, YI, HI, and TU performed the mouse experiments. SN, TS, AO, and KM performed the human cell experiments. HA provided instructions. TY and KT supervised the project. All authors read and approved the final manuscript. 


\section{Consent for publication}

Not applicable.

\section{Ethics approval}

All research and animal care procedures were approved by the Tokyo Medical and Dental University Animal Care and Use Committee.

\section{Author details}

'Laboratory of Molecular Neuroscience, Medical Research Institute (MRI), Tokyo Medical and Dental University (TMDU), 1-5-45, Yushima, Bunkyo, Tokyo 113-8510, Japan. '2Laboratory of Recombinant Animals, MRI, TMDU, 2-3-10, Surugadai, Kanda, Chiyoda, Tokyo 101-0062, Japan. ${ }^{3}$ The Center for Brain Integration Research (CBIR), TMDU, Tokyo 113-8510, Japan. ${ }^{4}$ Department of Animal Risk Management, Chiba Institute of Science, 3 Shiomi-cho, Choshi, Chiba 288-0025, Japan. ${ }^{5}$ Department of Mathematical and Life Sciences, Graduate School of Science, Hiroshima University, 1-3-1, Kagamiyama, Higashi-Hiroshima, Hiroshima 739-8526, Japan. ${ }^{6}$ Department of Neurobiology, Institute of Biomedical and Health Sciences, Hiroshima University, 1-2-3, Kasumi, Minami-ku, Hiroshima 734-8553, Japan. ${ }^{7}$ Present address: McGovern Institute for Brain Research, Massachusetts Institute of Technology, 43 Vassar St., Cambridge, MA 02139, USA. ${ }^{8}$ Present address: Graduate School of Bioagricultural Sciences, Nagoya University, Furo-cho, Chikusa-ku, Nagoya 464-8601, Japan

\section{Received: 3 September 2016 Accepted: 22 November 2016}

\section{Published online: 28 November 2016}

\section{References}

1. Huang ZJ, Zeng H. Genetic approaches to neural circuits in the mouse. Annu Rev Neurosci. 2013;36:183-215.

2. Capecchi MR. Gene targeting in mice: functional analysis of the mammalian genome for the twenty-first century. Nat Rev Genet. 2005;6:507-12.

3. Sternberg SH, Doudna JA. Expanding the Biologist's Toolkit with CRISPRCas9. Mol Cell. 2015;58:568-74.

4. Doudna JA, Charpentier E. The new frontier of genome engineering with CRISPR-Cas9. Science. 2014;346:1258096.

5. Hsu PD, Lander ES, Zhang F. Development and Applications of CRISPR-Cas9 for Genome Engineering. Cell. 2014;157:1262-78.

6. Yang $H$, Wang $H$, Shivalila CS, Cheng AW, Shi L, Jaenisch R. One-step generation of mice carrying reporter and conditional alleles by CRISPR/ Cas-mediated genome engineering. Cell. 2013;154:1370-9.

7. Yang $\mathrm{H}$, Wang $\mathrm{H}$, Jaenisch $\mathrm{R}$. Generating genetically modified mice using CRISPR/Cas-mediated genome engineering. Nat Protoc. 2014;9:1956-68.

8. Aida T, Chiyo K, Usami T, Ishikubo H, Imahashi R, Wada $Y$, et al. Cloning-free CRISPR/Cas system facilitates functional cassette knock-in in mice. Genome Biol. 2015;16:87.

9. Skarnes WC. Is mouse embryonic stem cell technology obsolete? Genome Biol. 2015;16:109.

10. Zhang L, Jia R, Palange NJ, Satheka AC, Togo J, An Y, et al. Large genomic fragment deletions and insertions in mouse using CRISPR/Cas9. PLoS One. 2015:10:e0120396.

11. Aida T, Imahashi R, Tanaka K. Translating human genetics into mouse: the impact of ultra-rapid in vivo genome editing. Dev Growth Differ. 2014;56:34-45.

12. Singh $P$, Schimenti JC, Bolcun-Filas E. A mouse Geneticist's practical guide to CRISPR applications. Genetics. 2014;199:1-15.

13. Gong S, Zheng C, Doughty ML, Losos K, Didkovsky N, Schambra UB, et al. A gene expression atlas of the central nervous system based on bacterial artificial chromosomes. Nature. 2003:425:917-25.

14. Skarnes WC, Rosen B, West AP, Koutsourakis M, Bushell W, lyer V, et al. A conditional knockout resource for the genome-wide study of mouse gene function. Nature. 2011:474:337-42.

15. Lienert F, Lohmueller JJ, Garg A, Silver P. Synthetic biology in mammalian cells: next generation research tools and therapeutics. Nat Rev Mol Cell Biol. 2014;15:95-107.

16. Mao Z, Bozzella M, Seluanov A, Gorbunova V. DNA repair by nonhomologous end joining and homologous recombination during cell cycle in human cells. Cell Cycle. 2008;7:2902-6.

17. McVey M, Lee SE. MMEJ repair of double-strand breaks (director's cut): deleted sequences and alternative endings. Trends Genet. 2008;24:529-38.
18. Wang $H$, Yang $H$, Shivalila CS, Dawlaty MM, Cheng AW, Zhang F, et al One-step generation of mice carrying mutations in multiple genes by CRISPR/Cas-mediated genome engineering. Cell. 2013;153:910-8.

19. Bae S, Kweon J, Kim HS, Kim J-S. Microhomology-based choice of Cas9 nuclease target sites. Nat Methods. 2014;11:705-6.

20. Nakade S, Tsubota T, Sakane Y, Kume S, Sakamoto N, Obara M, et al. Microhomology-mediated end-joining-dependent integration of donor DNA in cells and animals using TALENs and CRISPR/Cas9. Nat Commun. 2014;5:5560.

21. Sakuma T, Nakade S, Sakane Y, Suzuki KT, Yamamoto T. MMEJ-assisted gene knock-in using TALENs and CRISPR-Cas9 with the PITCh systems. Nat Prot. 2015;11:118.

22. Sakuma T, Takenaga M, Kawabe $Y$, Nakamura T, Kamihira M, Yamamoto T. Homologous recombination-independent large gene cassette knock-in in CHO cells using TALEN and MMEJ-directed donor plasmids. Int J Mol Sci. 2015;16:23849-66

23. Hisano Y, Sakuma T, Nakade S, Ohga R, Ota S, Okamoto H, et al. Precise in-frame integration of exogenous DNA mediated by CRISPR/Cas9 system in zebrafish. Sci Rep. 2015:5:8841.

24. Tanaka KF, Matsui K, Sasaki T, Sano H, Sugio S, Fan K, et al. Expanding the repertoire of optogenetically targeted cells with an enhanced gene expression system. Cell Rep. 2012;2:397-406.

25. Kanemaru K, Sekiya H, Xu M, Satoh K, Kitajima N, Yoshida K, et al. In vivo visualization of subtle, transient, and local activity of astrocytes using an ultrasensitive Ca2+ indicator. Cell Rep. 2014;8:311-8.

26. Fenno LE, Mattis J, Ramakrishnan C, Hyun M, Lee SY, He M, et al. Targeting cells with single vectors using multiple-feature Boolean logic. Nat Methods. 2014;11:763-72.

27. Xiong $X$, Du Z, Wang Y, Feng Z, Fan P, Yan C, et al. 53BP1 promotes microhomology-mediated end-joining in G1-phase cells. Nucleic Acids Res. 2015;43:1659-70.

28. Heyer W-D, Ehmsen KT, Liu J. Regulation of homologous recombination in eukaryotes. Annu Rev Genet. 2010;44:113-39.

29. Mashimo T, Kaneko T, Sakuma T, Kobayashi J, Kunihiro Y, Voigt B, et al. Efficient gene targeting by TAL effector nucleases coinjected with exonucleases in zygotes. Sci Rep. 2013:3:1253.

30. Sakuma T, Nishikawa A, Kume S, Chayama K, Yamamoto T. Multiplex genome engineering in human cells using all-in-one CRISPR/Cas9 vector system. Sci Rep. 2014;4:5400

31. Ng SY, Gunning P, Eddy R, Ponte P, Leavitt J, Shows T, et al. Evolution of the functional human beta-actin gene and its multi-pseudogene family: conservation of noncoding regions and chromosomal dispersion of pseudogenes. Mol Cell Biol. 1985;5:2720-32.

32. Cradick TJ, Qiu P, Lee CM, Fine EJ, Bao G. COSMID: a Web-based tool for identifying and validating CRISPR/Cas Off-target sites. Mol Ther Nucleic Acids. 2014;3:e214

33. Izu Y, Sun M, Zwolanek D, Veit G, Williams V, Cha B, et al. Type XII collagen regulates osteoblast polarity and communication during bone formation. J Cell Biol. 2011:193:1115-30.

34. Decottignies A. Microhomology-mediated end joining in fission yeast is repressed by Pku70 and relies on genes involved in homologous recombination. Genetics. 2007;176:1403-15.

35. Fiorentini P, Huang KN, Tishkoff DX, Kolodner RD, Symington LS. Exonuclease of Saccharomyces cerevisiae functions in mitotic recombination in vivo and in vitro. Mol Cell Biol. 1997:17:2764-73.

36. Certo MT, Gwiazda KS, Kuhar R, Sather B, Curinga G, Mandt T, et al. Coupling endonucleases with DNA end-processing enzymes to drive gene disruption. Nat Methods. 2012;9:973-5.

37. Miura H, Gurumurthy CB, Sato T, Sato M, Ohtsuka M. CRISPR/Cas9-based generation of knockdown mice by intronic insertion of artificial microRNA using longer single-stranded DNA. Sci Rep. 2015;5:12799.

38. Chari R, Mali P, Moosburner M, Church GM. Unraveling CRISPR-Cas9 genome engineering parameters via a library-on-library approach. Nat Meth. 2015:12:823-6.

39. Srivastava M, Nambiar M, Sharma S, Karki SS, Goldsmith G, Hegde M, et al. An inhibitor of nonhomologous end-joining abrogates double-strand break repair and impedes cancer progression. Cell. 2012;151:1474-87.

40. Maruyama T, Dougan SK, Truttmann MC, Bilate AM, Ingram JR, Ploegh $\mathrm{HL}$. Increasing the efficiency of precise genome editing with CRISPRCas9 by inhibition of nonhomologous end joining. Nat Biotechnol. 2015;33:538-42 
41. Chu VT, Weber T, Wefers B, Wurst W, Sander S, Rajewsky K, et al. Increasing the efficiency of homology-directed repair for CRISPR-Cas9-induced precise gene editing in mammalian cells. Nat Biotechnol. 2015;33:543-8.

42. Yu C, Liu Y, Ding S, Qi LS, Yu C, Liu Y, et al. Small molecules enhance CRISPR genome editing in small molecules enhance CRISPR genome editing in pluripotent stem cells. Cell Stem Cell. 2015;16:142-7.

43. Jayathilaka K, Sheridan SD, Bold TD, Bochenska K, Logan HL, Weichselbaum RR, et al. A chemical compound that stimulates the human homologous recombination protein RAD51. Proc Natl Acad Sci U S A. 2008;105:15848-53.

44. Pinder J, Salsman J, Dellaire G. Nuclear domain 'knock-in' screen for the evaluation and identification of small molecule enhancers of CRISPR-based genome editing. Nucleic Acids Res. 2015;43:9379-92.

45. Song J, Yang D, Xu J, Zhu T, Chen YE, Zhang J. RS-1 enhances CRISPR/Cas9and TALEN-mediated knock-in efficiency. Nat Commun. 2016;7:10548.

46. Ran FA, Hsu PD, Wright J, Agarwala V, Scott DA, Zhang F. Genome engineering using the CRISPR-Cas9 system. Nat Protoc. 2013;8:2281-308.

47. Sakuma T, Ochiai H, Kaneko T, Mashimo T, Tokumasu D, Sakane Y, et al. Repeating pattern of non-RVD variations in DNA-binding modules enhances TALEN activity. Sci Rep. 2013;3:3379.

48. Ochiai H, Fujita K, Suzuki K, Nishikawa M, Shibata T, Sakamoto N, et al. Targeted mutagenesis in the sea urchin embryo using zinc-finger nucleases. Genes Cells. 2010;15:875-85.

49. Mussolino C, Morbitzer R, Lütge F, Dannemann N, Lahaye T, Cathomen T. A novel TALE nuclease scaffold enables high genome editing activity in combination with low toxicity. Nucleic Acids Res. 2011;39:9283-93.

50. Aida T, Yoshida J, Nomura M, Tanimura A, lino Y, Soma M, et al. Astroglial glutamate transporter deficiency increases synaptic excitability and leads to pathological repetitive behaviors in mice. Neuropsychopharmacology. 2015:40:1569-79.

51. Shaner NC, Lambert GG, Chammas A, Ni Y, Cranfill PJ, Baird MA, et al. A bright monomeric green fluorescent protein derived from Branchiostoma lanceolatum. Nat Methods. 2013;10:407-9.

\section{Submit your next manuscript to BioMed Central} and we will help you at every step:

- We accept pre-submission inquiries

- Our selector tool helps you to find the most relevant journal

- We provide round the clock customer support

- Convenient online submission

- Thorough peer review

- Inclusion in PubMed and all major indexing services

- Maximum visibility for your research

Submit your manuscript at www biomedcentral.com/submit
O Biomed Central 